\title{
Tactile Resolution: Peripheral Neural Mechanisms Underlying the Human Capacity to Determine Positions of Objects Contacting the Fingerpad
}

\author{
H. E. Wheat, A. W. Goodwin, and A. S. Browning \\ Department of Anatomy and Cell Biology, University of Melbourne, Parkville Victoria 3052, Australia
}

\begin{abstract}
We measured the ability of humans to discriminate the positions of spherical objects passively contacting the fingerpad. The discrimination threshold averaged $0.55 \mathrm{~mm}$ for a moderately curved sphere (radius $5.80 \mathrm{~mm}$ ) and decreased to $0.38 \mathrm{~mm}$ for a more curved sphere (radius 1.92 $\mathrm{mm}$ ); since the receptor density is about 1 per $\mathrm{mm}^{2}$, these values are substantially smaller than those predicted by the sampling theorem (referred to as hyperacuity). To elucidate the underlying neural mechanisms, responses to the same spheres and random sequences of stimuli were recorded from single Merkel afferents (SAls) and Meissner afferents (RAs) in anesthetized monkeys. For multiple applications of identical stimuli, coefficients of variation of responses were around $3 \%$. Profiles of responses across the SAl population were "hill-shaped." A change in position of the stimulus on the skin resulted in a matching shift of the profile, evident over the whole profile for the more curved sphere but only at the skirts for the less curved sphere. The shift in response proflles, relative to the standard deviations, increased as the change in position increased, and was more reliable for the more curved sphere. Responses were measured over four time frames: $0.2,0.3$, 0.5 , and $1.0 \mathrm{sec}$. Although responses increased with an increase in integration time, so, too, did their standard deviations, so that signal-to-noise ratios or the resolution in the SAI population was about the same at $0.2 \mathrm{sec}$ as at 1.0 sec. Only half the RAs responded; responses were small, but signalled reliable information about the position of the stimulus.
\end{abstract}

[Key words: tactile resolution, cutaneous mechanoreceptors, hyperacuity, position discrimination, peripheral nerve, monkey fingerpad]

When humans use their hands to manipulate and explore objects in the environment, precise information about the objects is relayed to the brain by the cutaneous mechanoreceptors. Early experiments comparing tactile acuity across the body surface, starting with Weber's measurement of the two-point limen in 1835 , showed that resolution was highest on the fingertips where its limit was of the order of a few millimeters (see Weinstein, 1968). The fingertips are also the region with the highest innervation density, estimated at 0.70 per $\mathrm{mm}^{2}$ for fibers innervaling

\footnotetext{
Received Jan. 17, 1995; revised March 16, 1995; accepled March 31, 1995.

This research was supported by a grant from the National Health and Medical Research Council of Australia.

Correspondence should be addressed to A. W. Goodwin at the above address

Copyright (C) 1995 Society for Neuroscience $0270-6474 / 95 / 155582-14 \$ 05.00 / 0$
}

the Merkel complexes and 1.41 per $\mathrm{mm}^{2}$ for fibers innervating the Meissner corpuscles (Johansson and Vallbo, 1979). More recently, in a variety of more controlled tasks, much greater resolution has been shown; for example, humans can discriminate a $50 \mu \mathrm{m}$ change in the spatial period of a grating (Morley et al., 1983). In some of these studies, recordings from primary afferent fibers in monkeys have clarified the neural mechanisms underlying the human performance (Phillips and Johnson, 1981).

Information about the position of an object on the skin is of crucial importance during fine manipulations. The classical measure for this attribute of the stimulus is the "error of localization," and it has becn known for a long time that performance here is superior to that suggested by the two-point limen (Dallenbach, 1932; Zigler, 1935). However, these early experiments were not well controlled, and the figure for resolution on the fingertip of nearly $2 \mathrm{~mm}$ (Dallenbach, 1932; Weinstein, 1968) is not consistent with the precise manipulative capacities of humans nor with more recent measures like grating resolution. In a well controlled study, Loomis (1979) found that his three subjects could localize stimuli with a resolution better than $0.2 \mathrm{~mm}$. There are other tasks, like vernier acuity, where resolution exceeds expectations based on classical measures or expectations based on the known innervation density of the fingerpads (Loomis and Collins, 1978; Loomis, 1979). The term hyperacuity has been used for such performance; there have been many psychophysical studies of this phenomenon in the visual system but relatively few neurophysiological investigations of the underlying mechanisms (Westheimer, 1975, 1981; Lee et al., 1993). In the tactile system, we are not aware of any studies that explicitly address the problem of how position information is relayed by the cutaneous afferents. In the experiments reported here, we have tackled the problem by measuring the human ability to discriminate the positions of spherical surfaces applied passively to the fingerpad. Using a nearly identical stimulus protocol, we characterized the responses in the populations of digital nerve fibers in monkeys in order to elucidate the neural mechanisms underlying the human performance.

\section{Materials and Methods}

The stimuli used in these experiments were plastic objects with spherically curved surfaces. These were applied passively to an immobilized fingerpad by a stimulator that has been described previously (Goodwin et al., 1991). Two spheres were used and were characterized either by their radii (in millimeters) or by their curvatures (in units $\mathrm{m}^{-1}$ ); curvature is the reciprocal of the radius and is a measure that is often more convenient. The more curved surface had a radius of $1.92 \mathrm{~mm}$ (curvature $521 \mathrm{~m}^{-1}$ ) and the less curved surface had a radius of $5.80 \mathrm{~mm}$ (curvature $172 \mathrm{~m}^{-1}$ ). A balanced beam, moved by gravity, lowered the stimuli onto the skin. The contact force was set by a counterbalance 
weight on the beam and was calibrated by an electronic balance; the resolution was about $0.1 \mathrm{gm}$ wt $\left(9.8 \times 10^{4} \mathrm{~N}\right)$. Motion of the beam was controlled by a rotary damper so that stimuli contacted the skin at a constant velocity of about $20 \mathrm{~mm} / \mathrm{sec}$. The beam was attached to an orthogonal pair of $(x-y)$ micrometers, fitted with dial indicators, so that the position of the stimulus could be altered rapidly with a resolution of $0.01 \mathrm{~mm}$.

Psychophysics. Subjects were seated comfortably behind a curtain. With the arm supinated, the index finger of their dominant hand was secured in a bed of plasticine (modeling clay) by attaching BLU-TACK (Bostik, Australia) to the back of the finger. The stimulator was used to apply spherically curved surfaces to the central part of the fingerpad the finger was positioned so that the applied force, at the point of contact, was normal to the skin. The stimulator was positioned $2 \mathrm{~mm}$ above the skin and the contact force was set at $40 \mathrm{gm}$ wt $(0.392 \mathrm{~N})$. To apply the stimulus, an electronic signal energized a relay that released the beam. The motion of the stimulus was monitored on an oscilloscope; after the stimulus had been in contact with the skin for about $1 \mathrm{sec}$, the experimenter raised it off the skin and reengaged the beam. A forced choice paradigm was used to measure the ability of human subjects to discriminate the position of the sphere on the fingerpad. Each trial consisted of a pair of stimuli. The first stimulus in the pair was the standard and was always applied at the same position on the fingerpad. The second in the pair was the comparison stimulus; it was applied either at the same position as the standard, in which case the two stimuli were the same $\left(S_{s}\right)$, or it was applied at some distance proximal to the standard position, in which case the 2 stimuli were different $\left(S_{d}\right)$. The distance $d$ was varied along an axis parallel to the long axis of the finger The time sequence of the stimuli is shown in Figure $1 A$; the time interval between pairs in a trial was about $2 \mathrm{sec}$, and the time interval between trials was about $5 \mathrm{sec}$. The subject's task was to respond that the stimuli were the same $\left(\mathrm{R}_{\mathrm{s}}\right)$ or that they were different $\left(\mathrm{R}_{\mathrm{d}}\right)$. Feedback was given after every trial. There were two series of experiments. In the first series, the radius of the surface was $5.80 \mathrm{~mm}$ (curvature 172 $\mathrm{m}^{-1}$ ) and the distance $d$ had one of the six values $0.1,0.2,0.3,0.5,0.7$, or $1.0 \mathrm{~mm}$. In the second series, the radius of the surface was $1.92 \mathrm{~mm}$ (curvature $521 \mathrm{~m}^{-1}$ ) and $d$ had one of the six values $0.05,0.1,0.2,0.3$, 0.5 , or $0.7 \mathrm{mum}$. The order of data collection was as follows. For the first series, a number of sessions were used to train the subjects in the task and, when their performance had stabilized, data were collected for 10 sessions. On each experiment day a block of data was collected for each of the six distances $d$. These six blocks were presented in a random order. Within each block there were 20 trials consisting of 10 $S_{\mathrm{s}}$ and $10 \mathrm{~S}_{\mathrm{d}}$ interspersed in a random order. The same pattern was repeated for the second series.

Neural recording. Responses were recorded from single mechanoreceptive afferent fibers innervating the fingerpads of anesthetized monkeys; these were isolated by microdissection of their median nerves. The techniques are standard and have been described in detail previously (Goodwin and Morley, 1987; Goodwin et al., 1995); only a brief description is given here. An intraperitoneal catheter was used for fluid replacement and for regular doses of sodium pentobarbitone to maintain surgical anesthesia. Rectal temperature was monitored, and was maintained at $37^{\circ} \mathrm{C}$ by a heating blanket. Respiration rate, heart rate, blood pressure, end tidal carbon dioxide levels and oxygen saturation levels were also monitored. Antibiotics were administered during and at the end of the experiment.

Slowly adapting type I afferents arising from Merkel complexes (SAIs) and rapidly adapting afferents arising from Meissner corpuscles (RAs) were identified by the well-established criteria of responses to static stimuli, thresholds to rapidly changing or vibrating stimuli, and sizes of receptive fields (Talbot et al., 1968; Vallbo and Johansson, 1984). SAIs and RAs were accepted for study only if their receptive fields were located on the central region of a fingerpad. Responses were recorded from 22 SAIs and 21 RAs isolated from 21 median nerves. Seven Macaca nemestrina and six Macaca fascicularis were used: for 11 monkeys the weights ranged from 3.0 to $7.0 \mathrm{~kg}$, and two of the $M$. nemestrina were larger $(10.5$ and $12.0 \mathrm{~kg})$.

When a suitable afferent had been isolated, the center of the receptive field was estimated using a series of graded von Frey hairs. The finger was immobilized in a bed of plasticine with the nail secured by cotton thread, and the stimulator was positioned above the receptive field. The angle of the finger was such that the plane tangential to the fingerpad at the receptive field center was parallel to the experiment table and thus also to the plane of motion of the $x-y$ micrometers of the stimulator

\section{A. Timing - psychophysics}

Trial 1

Trial 2

Trial 20

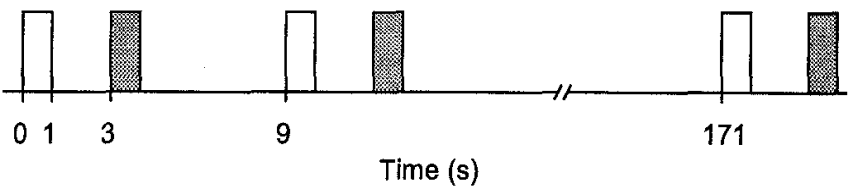

\section{B. Timing - neural recording}

Trial 1

Trial 2

Trial 13

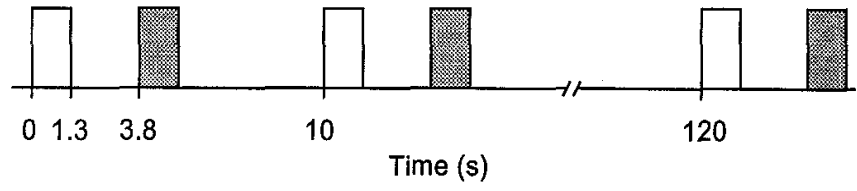

\section{Coordinate system}
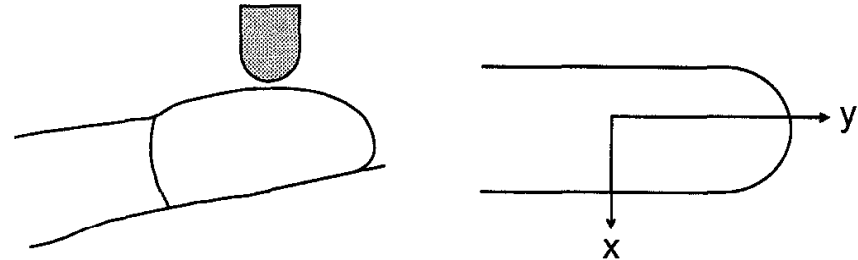

Figure 1. Time sequence of paired stimuli. Each trial consisted of a standard stimulus (open bar) followed by a comparison stimulus (filled bar). The standard was applied at one position on the skin, and the comparison was applied either at the same position $\left(S_{\mathrm{s}}\right)$ or at a second position a set distance from the first position $\left(S_{d}\right)$. A, For psychophysics, a block of data consisted of 20 trials with time intervals as indicated. $B$, For neural recording, there were 13 trials per block with similar time intervals. $C$, The monkey's finger was positioned so that the plane tangential to the skin, at the receptive field center, was parallel to the base of the stimulator. The y-axis, parallel to the long axis of the finger, and the $\mathrm{x}$-axis, at right angles to it, passed through the receptive field center.

(see Fig. 1C). The center of the receptive field was then determined more precisely as follows. Using a surface with a radius of $1.44 \mathrm{~mm}$ (curvature of $694 \mathrm{~m}^{-1}$ ) the stimulus was applied, at a contact force of $15 \mathrm{gm} \mathrm{wt}(0.147 \mathrm{~N})$, to points separated by $0.5 \mathrm{~mm}$ along two orthogonal axes through the estimated receptive field center; one axis was parallel to the long axis of the finger and the other was at right angles to it. At each point the total response over $1 \mathrm{sec}$ was measured to find the point of maximum response. The process was repeated around this point to confirm the center of the receptive field. Position on the skin was defined by Cartesian coordinates with the $y$-axis parallel to the long axis of the finger and pointing distally and the $\mathrm{x}$-axis at right angles to it; the origin was at the center of the receptive field (Fig. 1C).

The two curved surfaces used in the human experiments were also used on the monkeys. A rotary hub on the stimulator allowed either of the two surfaces to be selected rapidly. The monkey's hand was mounted on a micrometer fitted with a dial indicator with a resolution of 0.01 $\mathrm{mm}$. The hand was raised until the skin just touched the surface and then was lowered $0.5 \mathrm{~mm}$ so that when the stimulator was activated, the selected surface moved through the $0.5 \mathrm{~mm}$ gap to contact the skin. Contact force was set at $15 \mathrm{gm}$ wt $(0.147 \mathrm{~N})$.

For each afferent studied, the protocol of data collection was as follows. One of the two spheres (radius $5.80 \mathrm{~mm}$ or $1.92 \mathrm{~mm}$ ) was selected at random and positioned at the receptive field center. A block of data consisting of 13 trials was collected with a time sequence chosen to parallel that used in the psychophysical studies. Each trial consisted of the standard stimulus positioned at the center of the receptive field followed by the comparison stimulus, which was either at the same point $\left(\mathrm{S}_{\mathrm{s}}\right)$ or at a point some distance from it $\left(\mathrm{S}_{\mathrm{d}}\right)$. The distance was fixed at one of three values, $0.2,0.5$, or $0.8 \mathrm{~mm}$, and the displacement was 

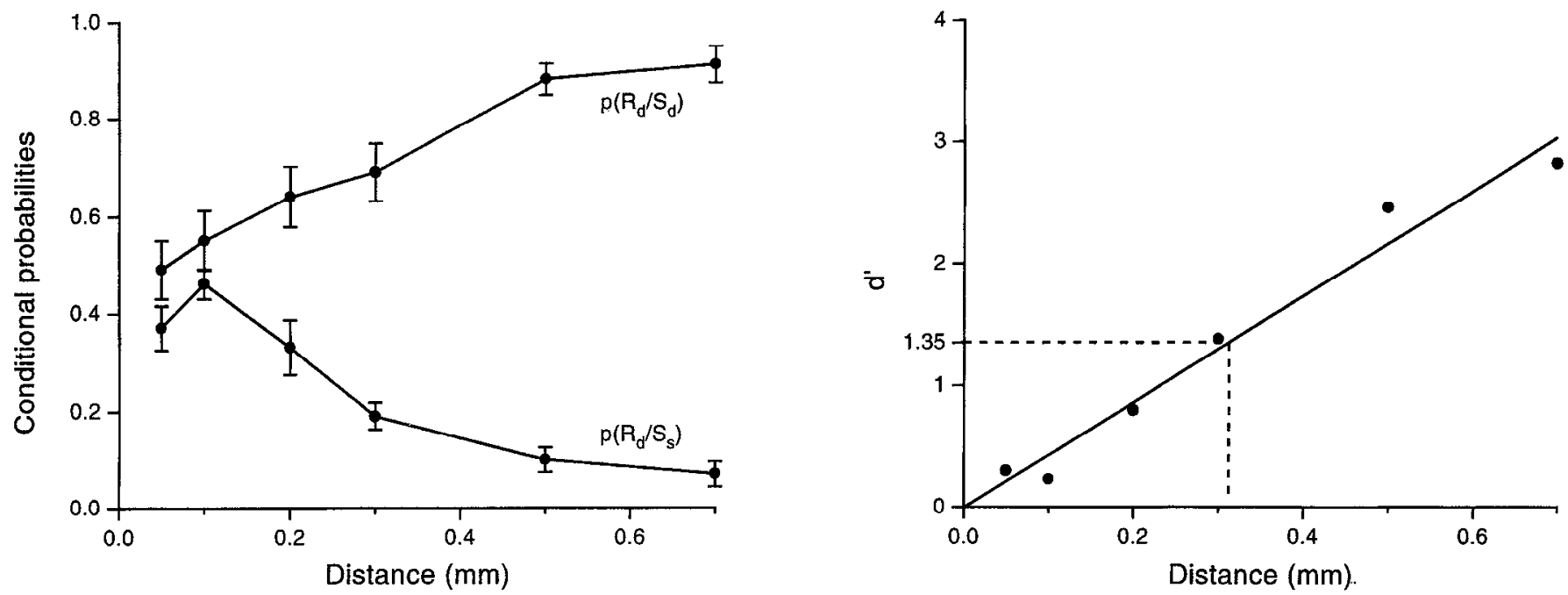

Figure 2. Performance of subject $\mathrm{S}_{5}$ discriminating the position of a surface with radius $1.92 \mathrm{~mm}$ (curvature $521 \mathrm{~m}^{-1}$ ). Left, Data points show mean values $( \pm \mathrm{SEM})$ of the conditional probabilities $p\left(\mathrm{R}_{\mathrm{d}} / \mathrm{S}_{\mathrm{d}}\right)$ and $p\left(\mathrm{R}_{\mathrm{d}} / \mathrm{S}_{\mathrm{s}}\right), n=10$. Right, Data points show values of the discriminatory separation index $d^{\prime}, 200$ observations for each point. The regression line was used to determine the difference limen $(0.31 \mathrm{~mm})$, which corresponds to $d^{\prime}=$ 1.35 .

always in a proximal direction along a line parallel to the long axis of the finger. The first trial in the block was $S_{s}$ and the remaining 12 consisted of $6 \mathrm{~S}_{\mathrm{s}}$ and $6 \mathrm{~S}_{\mathrm{d}}$ in a random order. An electronic pulse energized the relay on the stimulator, releasing the beam and lowering the surface onto the skin; after $1.3 \mathrm{sec}$ the relay released and the experimenter raised the surface off the skin. The second (comparison) stimulus in a trial commenced $3.8 \mathrm{sec}$ after the first (standard) stimulus commenced, and successive trials occurred every 10 seconds (see Fig. $1 B$ ). A contact time of $1.3 \mathrm{sec}$ was used to ensure a response of at least $1 \mathrm{sec}$ duration for analysis. There was some variability in the time taken for the experimenter to raise the stimulator beam. Thus, the occurrence of the second stimulus in a trial was set to ensure an interstimulus interval of at least $2 \mathrm{sec}$. Two more blocks of data were collected for the remaining two values of $d$; the order of the three values of the distance was chosen randomly. Following this, the height of the stimulator above the skin was checked and, if nccessary, reset to $0.5 \mathrm{~mm}$, and the procedure was repeated for the second sphere. The stimulator was then located at a different position in the receptive field and the whole process was repeated. As many positions as possible were used; these lay on a $0.5 \mathrm{~mm}$ grid defined by the $\mathrm{x}$ - and $\mathrm{y}$-axes passing through the receptive field center. The limiting factors were the position of the interphalangeal crease (proximal limit) and the curvature of the fingerpad (distal, medial, and lateral limits).

\section{Results}

\section{Psychophysics}

Subjects were presented with pairs of stimuli. The first in the pair, the standard, was always at the same position on the fingerpad and the sccond, the comparison, was either at the same position $\left(S_{s}\right)$ or at a different position $\left(S_{d}\right)$ proximal to the first position. Subjects responded that the stimuli were the same $\left(\mathbf{R}_{s}\right)$ or different $\left(R_{d}\right)$. At the end of each session, the conditional

\section{A. Radius $5.80 \mathrm{~mm}$ (curvature $172 \mathrm{~m}^{-1}$ )}

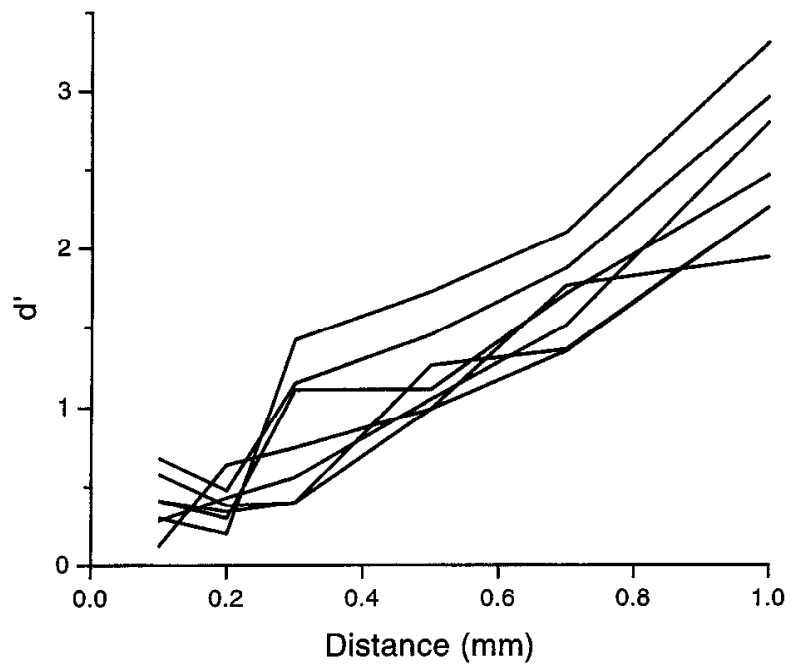

\section{B. Radius $1.92 \mathrm{~mm}$ (curvature $521 \mathrm{~m}^{-1}$ )}

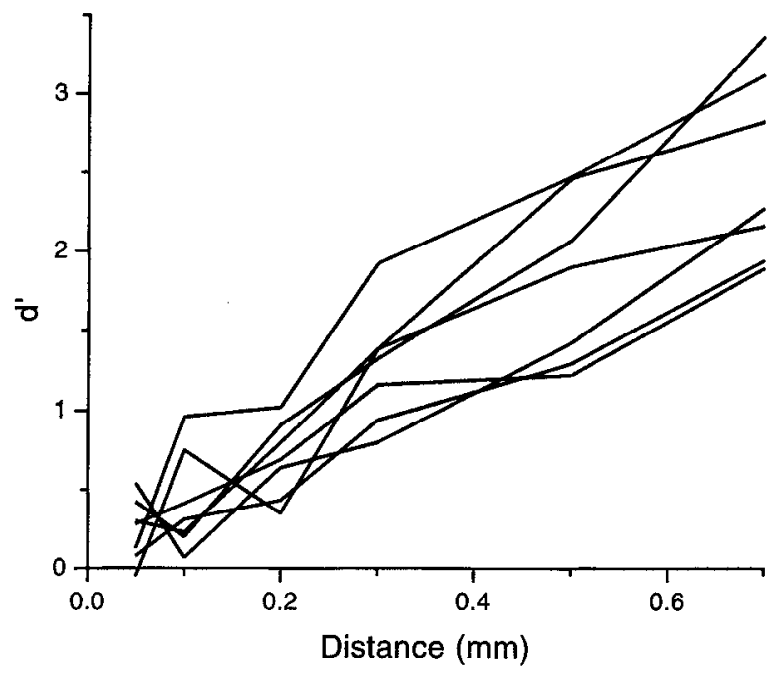

Figure 3. Discrimination performance for seven naive subjects. $A$, Series 1 . Spherical surface had a radius of $5.80 \mathrm{~mm}$ (curvature $172 \mathrm{~m}^{-1}$ ). Distances between standard and comparison stimuli for $S_{\mathrm{d}}$ were $0.1,0.2,0.3,0.5,0.7$, or $1.0 \mathrm{~mm}$. For each subject, the line joins values of $d$ ' at the six distances tested. $B$, Series 2 . Radius was $1.92 \mathrm{~mm}$ (curvature $521 \mathrm{~m}^{-1}$ ), separations were $0.05,0.1,0.2,0.3,0.5$, or $0.7 \mathrm{~mm}$. Note the difference in the distance scales for the two series. 
Table 1. Difference limens for position on the skin

\begin{tabular}{lll} 
Subject & $\begin{array}{l}\text { Series 1 } \\
\left(172 \mathrm{~m}^{-1}\right)\end{array}$ & $\begin{array}{l}\text { Series } 2 \\
\left(521 \mathrm{~m}^{-1}\right) \\
\mathrm{DL}(\mathrm{mm})\end{array}$ \\
\hline $\mathrm{S}_{1}$ & 0.63 & 0.32 \\
$\mathrm{~S}_{2}$ & 0.44 & 0.24 \\
$\mathrm{~S}_{3}$ & 0.56 & 0.48 \\
$\mathrm{~S}_{4}$ & 0.64 & 0.49 \\
$\mathrm{~S}_{5}$ & 0.42 & 0.31 \\
$\mathrm{~S}_{6}$ & 0.54 & 0.39 \\
$\mathrm{~S}_{7}$ & 0.62 & 0.44 \\
Mean & 0.55 & 0.38 \\
SEM & 0.034 & 0.036
\end{tabular}

Data are difference limens (DL) for seven naive subjects. In series 1, the radius of the surface was $5.80 \mathrm{~mm}$ (curvature $172 \mathrm{~m}^{-1}$ ), and in series 2, the radius was $1.92 \mathrm{~mm}$ (curvature $521 \mathrm{~m}^{-5}$ ). The mean DL of $0.55 \mathrm{~mm}$ for series 1 is significantly greater than the mean DL of $0.38 \mathrm{~mm}$ for series $2(p=0.00048$, one-tail paired $t$ test).

probabilities $p\left(\mathrm{R}_{\mathrm{d}} / \mathrm{S}_{\mathrm{d}}\right)$ and $p\left(\mathrm{R}_{\mathrm{d}} / \mathrm{S}_{\mathrm{s}}\right)$ were computed. The means and standard errors of these probabilities were used to determine when a sufficient number of sessions had been conducted. Figure 2 (left) shows a typical result; in this case, for one subject discriminating positions of the more curved sphere (radius 1.92 $\mathrm{mm}$ ). The low standard errors indicate that 10 sessions gave repeatable and statistically reliable observations. Using standard decision theory, a bias free measure of discrimination, $d^{\prime}$, was computed from the conditional probabilities over the 10 sessions (Johnson, 1980). The data points in Figure 2 (right) show $d^{\prime}$ values and the line shows the linear regression of the data points. The difference limen or discrimination threshold, defined by the distance giving a value for $d^{\prime}$ of 1.35 , was determined from the regression line. A $d^{\prime}$ value of 1.35 corresponds to a fractional correct score of $75 \%$ in the absence of bias (see Goodwin et al., 1991, for more detail on the rationale of the analysis). The $d^{\prime}$ functions for seven naive subjects, for both spheres, are shown in Figure 3. In all cases, the behavior of the subjects was similar, and $d^{\prime}$ increased as the distance between the standard and comparison stimuli increased. Values for the discrimination thresholds are given in Table 1. For all subjects, the difference limen for the less curved surface (radius $5.80 \mathrm{~mm}$, curvature $172 \mathrm{~m}^{-1}$ ) was considerably greater than for the more curved surface (radius $1.92 \mathrm{~mm}$, curvature $521 \mathrm{~m}^{-1}$ ). The mean value of $0.55 \mathrm{~mm}$ for the less curved surface is significantly greater than the mean of 0.38 inm for the more curved surface $(p=0.00048)$.

\section{Responses of single SAIs}

The spherical surfaces contacted the skin at a velocity of about $20 \mathrm{~mm} / \mathrm{sec}$, and their subsequent indentation of the skin was smoothed by the damper on the stimulator. The temporal characteristics of the SAI responses have been described by us pre-

\section{A. Response $-\mathrm{S}_{\mathrm{s}}$}

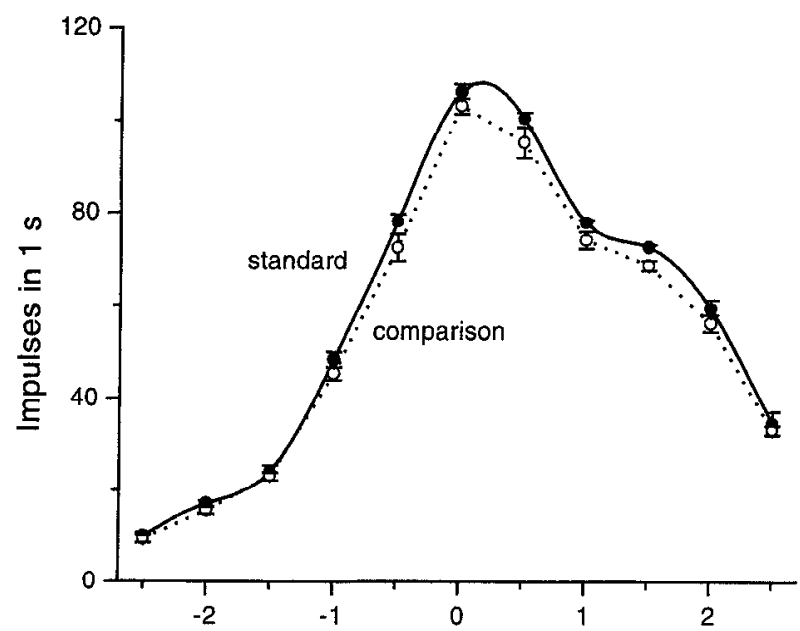

C. Difference in responses $-\mathrm{S}_{\mathrm{s}}$

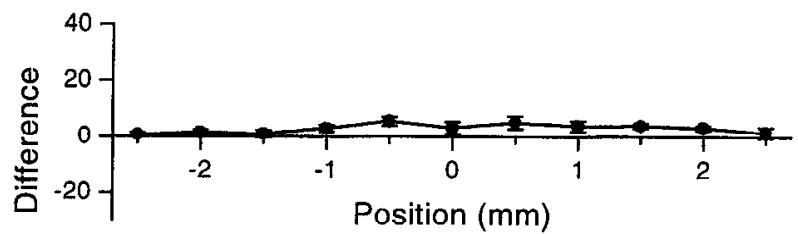

\section{B. Response $-S_{d}(0.5 \mathrm{~mm}$ distance $)$}

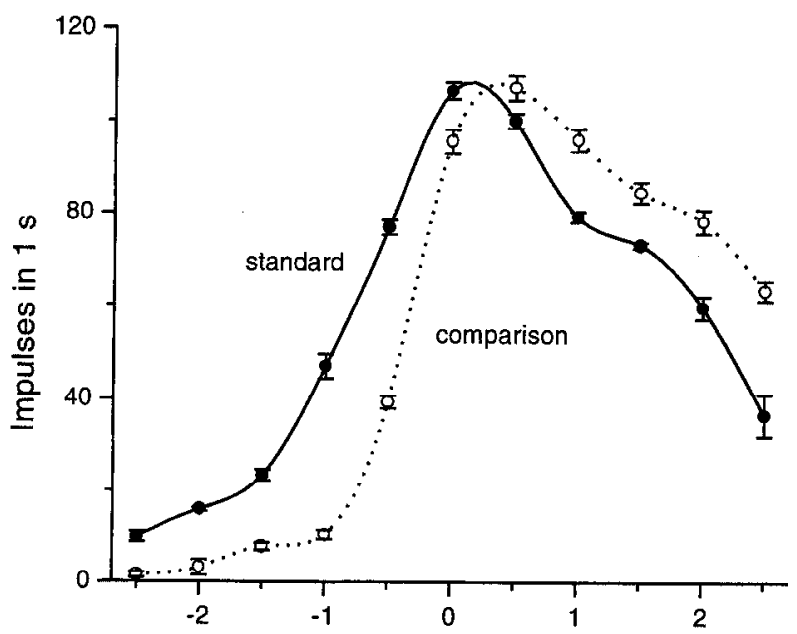

D. Difference in responses $-S_{d}$

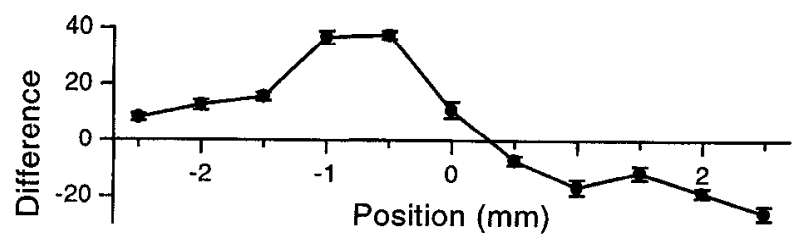

Figure 4. Responses to the standard and comparison stimuli when the standard was positioned at points, separated by $0.5 \mathrm{~mm}$, along a line through the center of the receptive field and parallel to the long axis of the finger. Abscissae show the position of the standard stimulus. The comparison stimulus was applied at the same position as the standard for trials in the left panel $\left(S_{s}\right)$, and $0.5 \mathrm{~mm}$ proximal to the standard in trials for the right panel $\left(S_{d}\right)$. $A$ and $B$ show responses over the first second (mean $\pm \mathrm{SD}$ ), $n=6$. $C$ and $D$ show differences in the responses (mean $\pm \mathrm{SD}$ ), $n=6$. The spherical surface had a radius of $1.92 \mathrm{~mm}$ (curvature $521 \mathrm{~m}^{-1}$ ). In $B$ and $D$, the proximal shift of the comparison stimulus results in a distal shift (positive y direction) of the profile (see Results). 
A. Radius $1.92 \mathrm{~mm}$, distance $0.2 \mathrm{~mm}$

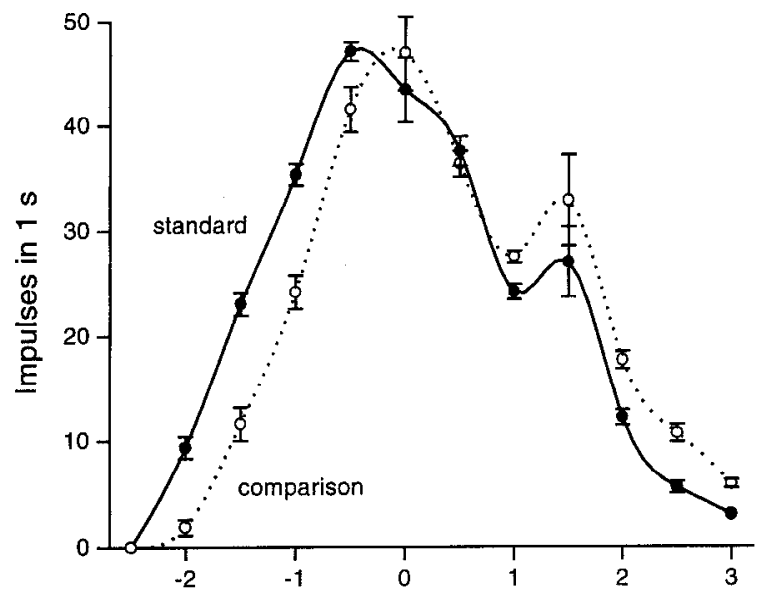

C. Radius $1.92 \mathrm{~mm}$, distance $0.5 \mathrm{~mm}$

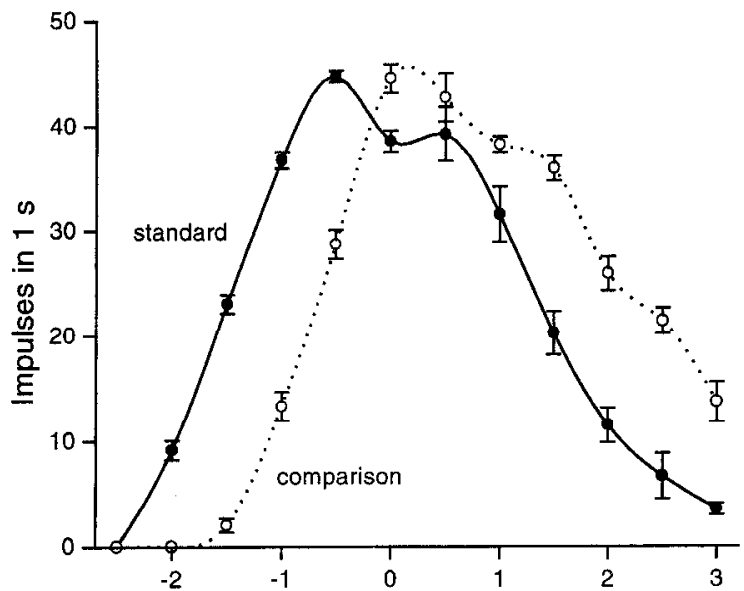

E. Radius $1.92 \mathrm{~mm}$, distance $0.8 \mathrm{~mm}$

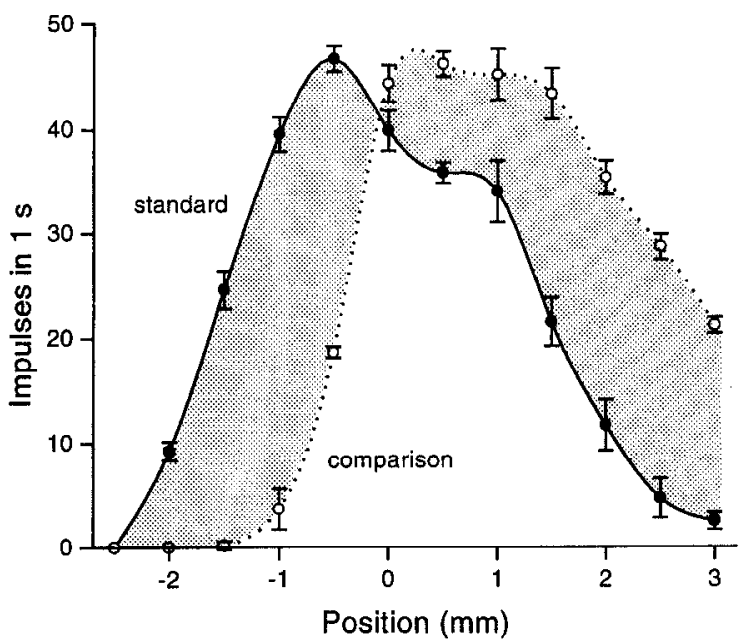

B. Radius $5.80 \mathrm{~mm}$, distance $0.2 \mathrm{~mm}$

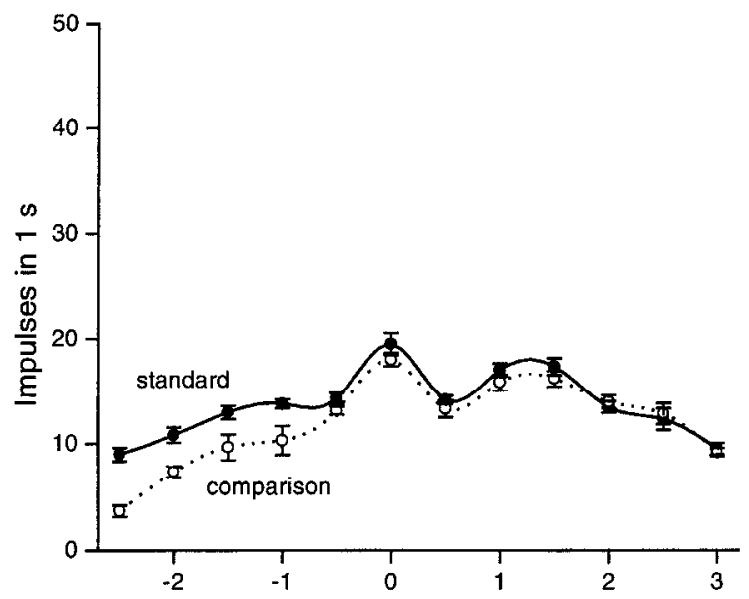

D. Radius $5.80 \mathrm{~mm}$, distance $0.5 \mathrm{~mm}$

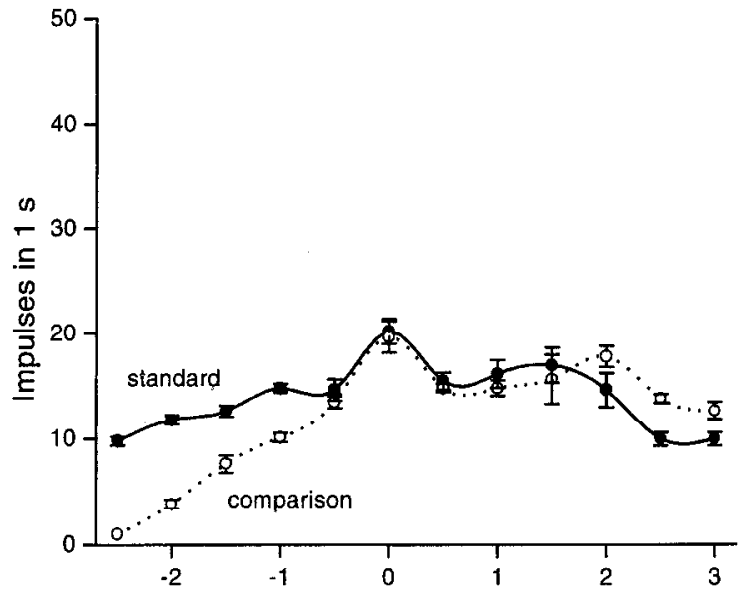

F. Radius $5.80 \mathrm{~mm}$, distance $0.8 \mathrm{~mm}$

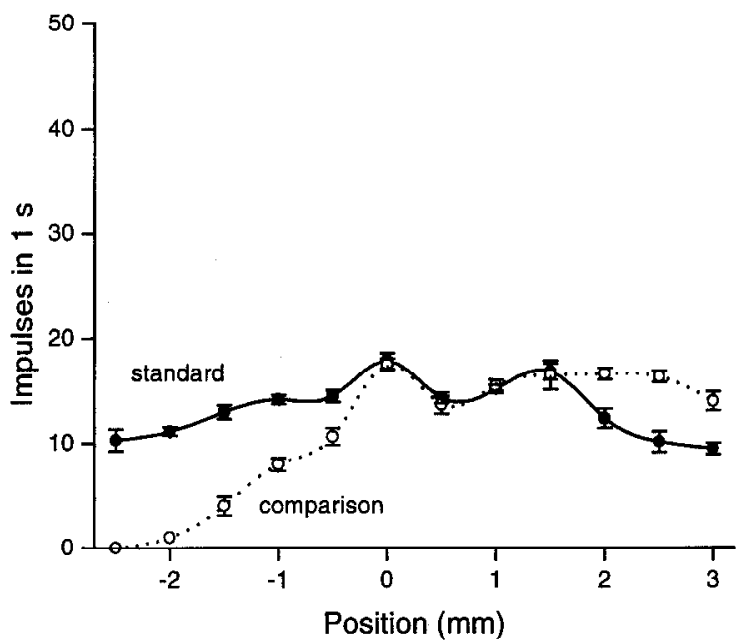

Figure 5. Responses for trials in which the comparison stimulus (broken line and open symbols) was applied proximal to the standard stimulus (solid line and filled symbols) $\left(\mathrm{S}_{4}\right)$. The standard was positioned at points, separated by $0.5 \mathrm{~mm}$, along a line through the center of the receptive field and parallel to the long axis of the finger; abscissae show the position of the standard. The distance between pairs of stimuli in a trial was 
viously (Goodwin et al., 1995). For most of the following analysis, the response measure used is the total number of action potentials evoked by the stimulus in the first second of response. This measure was chosen to correspond to the psychophysical measurements in humans, but other response characteristics are also explored. When the standard or the comparison stimuli were applied to the receptive field, the responses were variable. The ability of a population of afferents to discriminate two stimuli depends on the responses to the two stimuli differing in some way, and this difference must be greater than the inherent variability of the responses. There are many factors likely to influence the variability, such as random noise in the receptor mechanisms, variability in skin mechanics, interaction effects between standard and comparison stimuli, and interactions from preceding trials. It is to address this aspect of the problem that we chose to use random sequences of trials, some of which had the pair of stimuli in the same position $\left(\mathrm{S}_{\mathrm{s}}\right)$ and some of which had the pair in different positions $\left(S_{d}\right)$. The sequences used in the neural recording were similar to the sequences of stimuli used in the psychophysical experiments described above. Values chosen for the distance between standard and comparison stimuli spanned the critical range for human performance. The values were $0.8 \mathrm{~mm}$, which humans could discriminate for both spheres; $0.2 \mathrm{~mm}$, which could not be discriminated for either sphere; and $0.5 \mathrm{~mm}$, which could be discriminated for the more curved sphere but not for the less curved sphere.

To define the responses of fibers in a population of afferents with receptive field centers at varying distances from the stimulus, we repositioned the standard stimulus at a matrix of positions on the fingerpad. The aim here was to characterize, as completely as possible, the three-dimensional profile of responses with the emphasis on variability. Because of the timeconsuming nature of the data collection and the fact that receptive fields were not at the exact center of the monkey's fingerpad, which is considerably smaller than that in the human, certain compromises had to be made. As we have previously characterized the shape of the three-dimensional response profiles for the two standard stimuli, we were able to use this information to optimize the current experiments (Goodwin et al., 1995). First, we collected data from points, separated by $0.5 \mathrm{~mm}$, along the line through the center of the receptive field and parallel to the long axis of the finger (y-axis, see Fig. 1C). The number of points sampled on the $y$-axis $(x=0 \mathrm{~mm})$ was limited proximally by the interphalangeal crease and distally by the curvature of the fingerpad. Next, we collected data at $1 \mathrm{~mm}$ intervals along lines parallel to the $y$-axis and separated from it first by $1 \mathrm{~mm}$ $(x= \pm 1 \mathrm{~mm})$, then by $2 \mathrm{~mm}(x= \pm 2 \mathrm{~mm})$ and occasionally by $3 \mathrm{~mm}(\mathrm{x}= \pm 3 \mathrm{~mm})$. The limiting factor in the $\mathrm{x}$ direction was either the curvature of the fingerpad or time. At each point we repeated the same random sequences that we used at the center. As a check on consistency of responses, observations at the center of the receptive field were repeated a number of times during the experiment. The maximum number of points we were able to sample for one fiber was 35 which took $11.5 \mathrm{hr}$.

The curves in Figure 4 show the responses of a typical SAI to the sphere of radius $1.92 \mathrm{~mm}$ (curvature $521 \mathrm{~m}^{-1}$ ) positioned at successive points, separated by $0.5 \mathrm{~mm}$, along the $y$-axis. In the sequences of 13 trials, each trial commenced $10 \mathrm{sec}$ later than the previous one except for the first trial, which had an intertrial interval that was much longer and was variable, depending on the time taken to change surfaces, adjust the height above the skin, etc. For this reason, the first trial was excluded from the analysis, which therefore included six trials in which the standard and the comparison stimuli were in the same position $\left(\mathrm{S}_{\mathrm{s}}\right)$ and 6 trials in which they were in different positions $\left(S_{d}\right)$. Each group of six trials was combined to give a mean response and a standard deviation (a measure of the variability of the response). For the initial analysis, the response measure was the number of action potentials occurring during the first second of response. The trials in which both the standard and the comparison stimuli were presented at the same position in the receptive field are shown in Figure 4A. The filled symbols and solid line show responses (mean $\pm \mathrm{SD}$ ) for the standard stimulus and the open symbols and broken line show corresponding responses for the comparison stimulus. Figure $4 B$ shows the trials in which the comparison stimulus was applied $0.5 \mathrm{~mm}$ proximal to the standard at each position in the receptive field. The peak response for the comparison stimulus occurred when the standard was positioned distal to the center of the receptive field so that the proximal shift placed the stimulus at the receptive field center. Thus, in this figure, and in following figures, a proximal shift of the comparison stimulus is reflected in a distal shift of the profile or a shift in the positive y direction (see Fig. 1C). At a simple level it is clear that the profile for the comparison stimulus in Figure $4 B$ is shifted from the profile for the standard stimulus by about $0.5 \mathrm{~mm}$, and the standard deviations indicate that the two profiles are clearly distinguishable. Conversely, when the comparison stimulus was in the same position as the standard (Fig. 4A), there is no distinguishable shift between the profiles. These features are emphasized by the differences in responses to the standard and comparison stimuli shown in Figure 4, $C$ and $D$. When the stimuli were different $(D)$, the difference changes sign at $0.3 \mathrm{~mm}$, which is close to the distance between the two stimuli. When the stimuli were the same $(C)$, there is no change of sign; at each point the second response was slightly smaller than the first response, indicating a suppressive interaction between the two stimuli in a pair. This suppression was probably also present when the two stimuli were different, but it is swamped in Figure 4, $B$ and $D$, by the effect of the change in position.

The shift in response profiles is the underlying basis for changes in neural responses that allow the two positions of the stimulus to be distinguished. The manner in which this shift alters with different distances between the standard and comparison stimuli is elaborated in Figure 5 for another typical SAI. Profiles for the trials in which the two stimuli were different are shown for all three displacements: $0.2,0.5$, and $0.8 \mathrm{~mm}$. In the left and right panels, the surface had a radius of $1.92 \mathrm{~mm}$ (curvature $521 \mathrm{~m}^{-1}$ ) and $5.80 \mathrm{~mm}$ (curvature $172 \mathrm{~m}^{-1}$ ), respectively. For some afferents, like that in Figure 5, the peak response was not at the center of the receptive field as defined by symmetry; this was only apparent after the data had been analyzed. In such

$0.2 \mathrm{~mm}$ for $A$ and $B, 0.5 \mathrm{~mm}$ for $C$ and $D$, and $0.8 \mathrm{~mm}$ for $E$ and $F$. The spherical surfaces had a radius of $1.92 \mathrm{~mm}\left(\mathrm{curvature} 521 \mathrm{~m}^{-1}\right)$ for $A$, $C$, and $E$ and a radius of $5.80 \mathrm{~mm}$ (curvature $172 \mathrm{~m}^{-1}$ ) for $B, D$, and $F$. Responses (mean $\pm \mathrm{SD}, n=6$ ) are the number of impulses over the first second. Contact force was $15 \mathrm{gm}$ wt. The proximal shift of the comparison stimulus results in a distal shift (positive y direction) of the response profile. The shaded region in $E$ shows the area between the standard and comparison profiles. 
A. Radius $1.92 \mathrm{~mm}$ (curvature $521 \mathrm{~m}^{-1}$ )

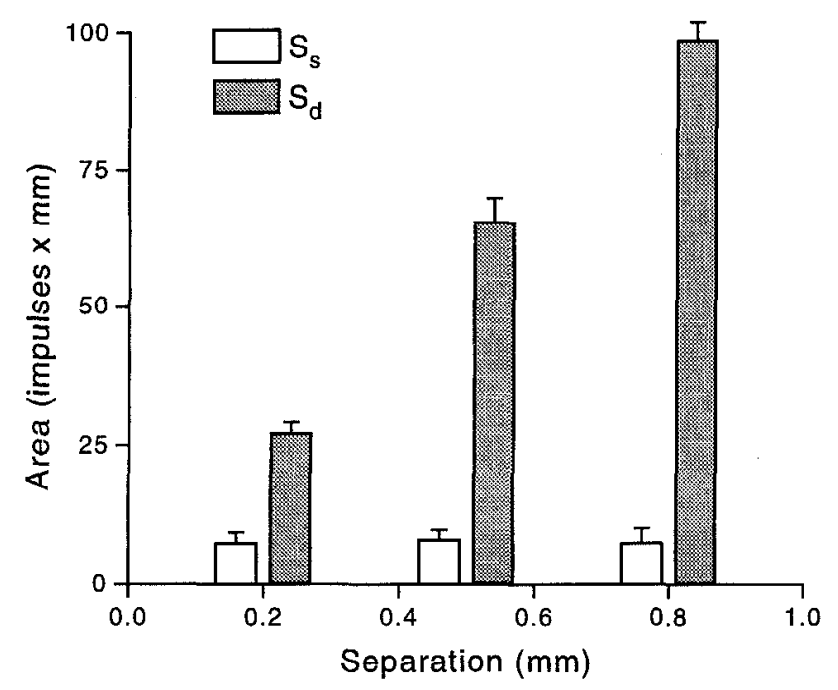

B. Radius $5.80 \mathrm{~mm}$ (curvature $172 \mathrm{~m}^{-1}$ )

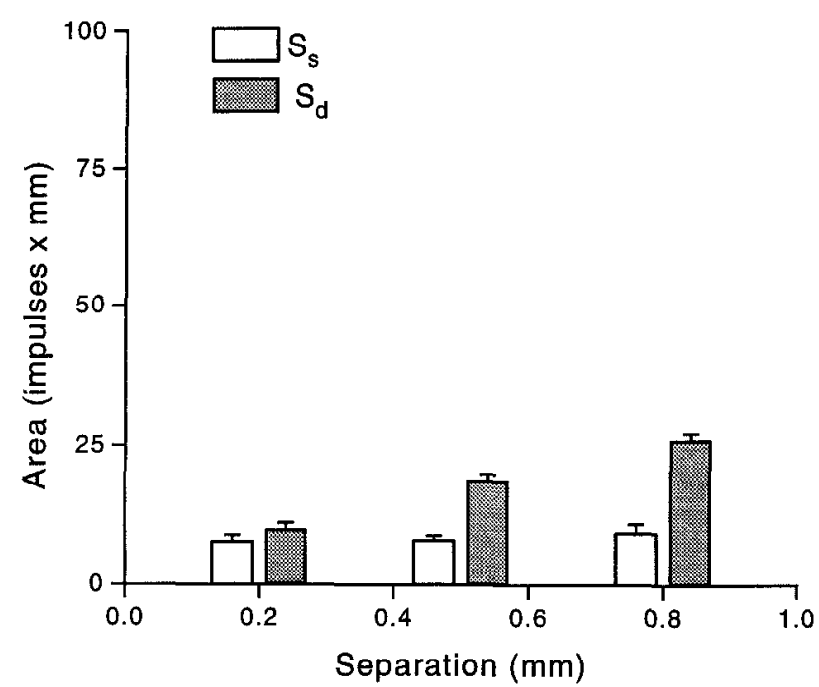

Figure 6. Area between the profile for the standard stimulus and the profile for the comparison stimulus for the fiber in Figure 5. Shaded bars and open bars show areas (mean + SD) when the standard and comparison stimuli were applied at different positions $\left(S_{d}\right)$ and at the same position $\left(\mathrm{S}_{\mathrm{s}}\right)$, respectively. The three separations between standard and comparison were $0.2,0.5$, and $0.8 \mathrm{~mm}$. A, Radius of spherical surface $1.92 \mathrm{~mm}$ (curvature $521 \mathrm{~m}^{-1}$ ). B, Radius $5.80 \mathrm{~mm}$ (curvature $172 \mathrm{~m}^{-1}$ ). The shaded bar for a separation of $0.8 \mathrm{~mm}$ in $A$ depicts the area of the shaded region in Figure $5 E$.

cases, the center was taken as the point of symmetry. For the more curved surface (left panel), the shift of the profiles increased as the distance between the standard and the comparison surfaces increased, and the magnitude of the shift matched the displacement between the stimuli. Moreover, it is evident that the magnitude of the shift relative to the noise level, indicated by the standard deviations, increased with an increase in displacement so that the certainty with which the profiles can be distinguished increases. For the less curved surface (right panel), the response profiles are lower in amplitude and flatter, as expected from our previous experiments (Goodwin et al., 1995). These profiles show similar shifts to those of the more curved surface but, because the profiles are flatter, the shift is only obvious around the skirts and not around the peaks. In addition, for the less curved surface, the shift in profile relative to the noise levels is less certain than for the more curved surface.

It is easier to appreciate the clarity of the shift if some measure of shift is extracted and compared to the variability. One simple and effective index of shift is the area between the profile for the standard stimulus and the profile for the comparison stimulus. The area, illustrated by the shaded region in Figure $5 E$, is easily approximated by summation of the component trapezoids. For each position (abscissa value) in Figure 5, there are six observations for each of the two curves. Thus, the area between the curves can be calculated six times, with the standard deviation of the area giving an indication of the reliability of the mean area. The shaded bars in Figure 6 show the areas for the six pairs of curves in Figure 5. For comparison, the open bars show the areas between the profiles for the standard and comparison stimuli when they were presented at the same position $\left(S_{\mathrm{s}}\right)$ on the skin. As seen in Figure 4, these areas $\left(\mathrm{S}_{\mathrm{s}}\right)$ result from a decrement in response due to stimulus interaction rather than from a shift. Nevertheless, they are instructive, since this effect may have contributed a small component to the shaded bars in Figure 6. Also, if a neural mechanism were based on a measure similar to this area, there would be a potential for false alarms in the case of $S_{s}$ or misses in the case of $S_{d}$ as, indeed, occurs in human discrimination. For the more curved stimulus (left panel), the area shown by the shaded bars increases as the separation between the standard and comparison stimuli increased, and is always considerably larger (relative to the standard deviations) than the area when the standard and comparison were in the same position (open bars). For the less curved stimulus (right pancl), the difference between the shaded $\left(S_{d}\right)$ and open $\left(S_{s}\right)$ bars is not as great as for the more curved stimulus, and for a separation of $0.2 \mathrm{~mm}$ the difference is of the same order as the standard deviations.

A simple comparison of these results with our psychophysical observations can be made by examining how these areas change as a function of the separation between the standard and comparison stimuli. Whether $S_{d}$ can be distinguished from $S_{\mathrm{s}}$ depends on whether the shaded bars in Figure 6 can be distinguished from the open bars. If the difference between these (difference between the area for $S_{d}$ and the area for $S_{s}$ ) is expressed in units of the standard deviation of the area, then the measure is analogous to the $d^{\prime}$ of our psychophysical experiments. In Figure 7 , the solid line and closed circles show the differences in areas in Figure $6 \mathrm{~A}$ divided by the average of the six standard deviations, and the solid line and closed triangles depict the same measure for Figure $6 B$. The dotted lines through the opcn circles or open triangles depict the psychophysical $d^{\prime}$ functions for the more curved and less curved sphere, respectively. There is broad agreement between the neural and psychophysical data in that, for both, the measure of discriminability increased with an increase in separation, and, for both, discrimination was better for the more curved sphere (radius $1.92 \mathrm{~mm}$, curvature $521 \mathrm{~m}^{-1}$ ) than for the less curved sphere (radius $5.80 \mathrm{~mm}$, curvature 172 $\mathrm{m}^{-1}$ ). Although this comparison is useful as an aid to understanding potential neural mechanisms, it should not be pushed to quantitative limits, since it takes no account of important fac- 


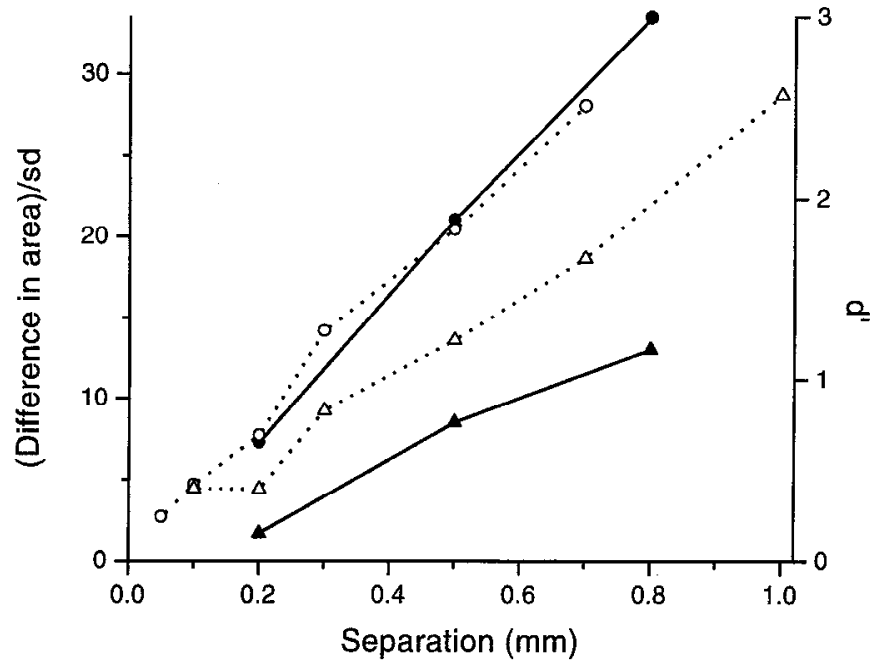

Figure 7. Comparison of psychophysical performance and neural responses. Dotted line and open circles or dotted line and open triangles show $d^{\prime}$ as a function of the separation between standard and comparison surfaces for the more curved sphere (radius $1.92 \mathrm{~mm}$, curvature $521 \mathrm{~m}^{-1}$ ) or the less curved sphere (radius $5.80 \mathrm{~mm}$, curvature 172 $\left.\mathrm{m}^{-1}\right)$, respectively. Solid line and closed circles or solid line and closed triangles show a corresponding neural measure extracted from Figure 6. This measure is the increase in the area between the profiles for the standard and comparison stimuli expressed in units of the standard deviation of the area.

tors like innervation density or interfiber variability (see Discussion).

When a spherical surface contacts the skin, a two-dimensional sheet of receptors is activated, resulting in a profile of responses in the innervating fibers that varies in both spatial dimensions. We have shown previously that the shape of this profile corresponds to the curvature of the surface and becomes higher and more peaked as the curvature increases; the profiles can be de- scribed by the function $a \exp \left(-b x^{2}-c y^{2}\right)$. For spherical surfaces with radii $1.92 \mathrm{~mm}$ (curvature $521 \mathrm{~m}^{-1}$ ) and $5.80 \mathrm{~mm}$ (curvature $172 \mathrm{~m}^{-1}$ ), the constants $a, b$, and $c$ were $1.67,0.23$, and 0.18 , and $0.86,0.02$, and 0.07 , respectively (Goodwin et al., 1995). The data from the experiments reported in the current manuscript are entirely consistent with our previous findings. When the position of the sphere on the skin changes, the whole profile shifts accordingly. We have illustrated this shift along the y-axis where we have concentrated our data collection, but the shift is seen over the complete profile for both dimensions on the skin.

\section{Responses combined for SAIs}

The profiles, illustrated so far for typical single fibers, were highly consistent for all the SAIs. Profiles for all fibers had the same shape and differed only by a scaling factor proportional to the sensitivity of the fiber. The homogeneity among the SAIs is demonstrated in Figure 8; data have been pooled for 13 fibers for which we collected extensive data along the y-axis. Profiles are shown for both spheres for the case when the standard and comparison stimuli were separated by $0.5 \mathrm{~mm}\left(\mathrm{~S}_{\mathrm{d}}\right)$. For each fiber, the responses were first normalized by dividing them by the normalizing factor for that fiber (the average response of the fiber over five central positions for both spheres). This eliminated the sensitivity of the fiber. $\Lambda s$ seen in the figure, the remaining underlying position-response profile is common to all fibers. Since one normalizing factor is used for each fiber, the relative heights of the profiles for the two spheres are preserved. The standard errors shown have a different meaning to the standard deviations shown in previous figures where they showed the variability of a single fiber to repeated applications of a stimulus. Here, the standard errors show the spread of the basic position-response profile among the different fibers in our sample. There is some asymmetry in the profiles, particularly for the less curved sphere, which may be due to the proximo-distal curvature of the fingerpad. Considering that our sample was

\section{A. Radius $1.92 \mathrm{~mm}$ (curvature $521 \mathrm{~m}^{-1}$ )}

\section{B. Radius $5.80 \mathrm{~mm}$ (curvature $172 \mathrm{~m}^{-1}$ )}
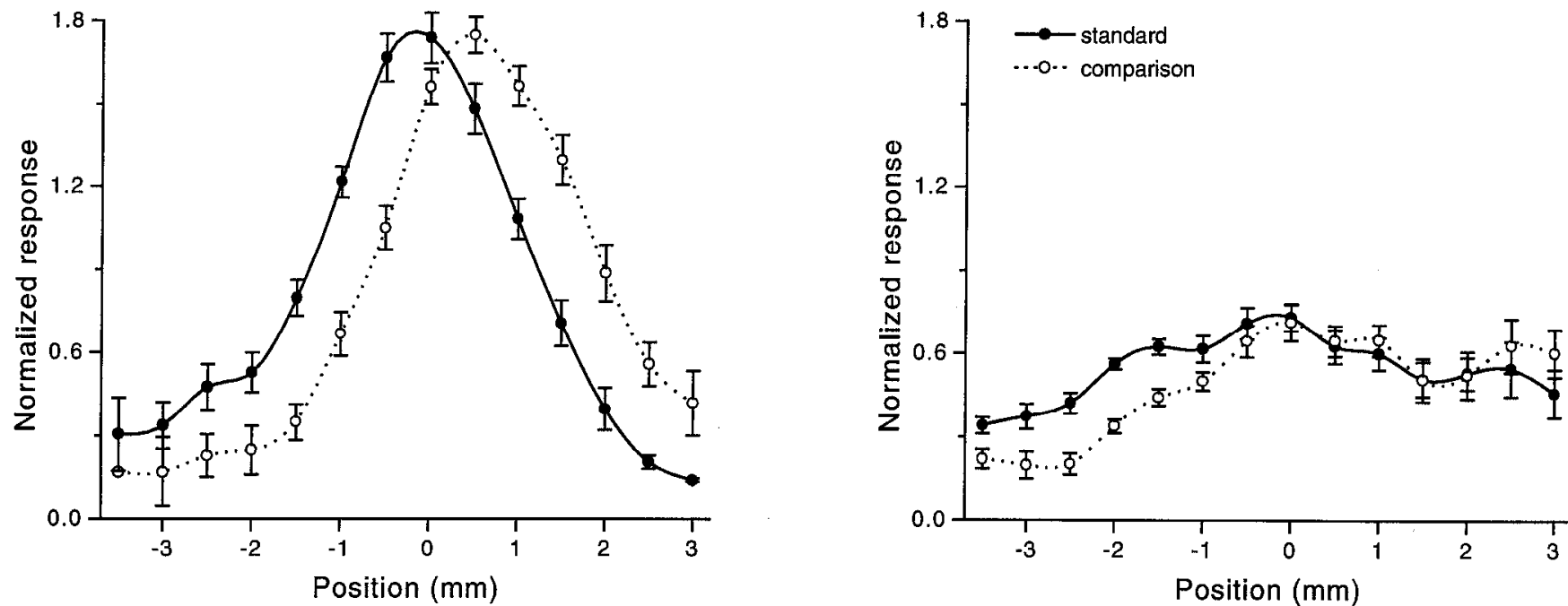

Figure 8. Normalized profiles for the sample of SAIs. Responses for each fiber were normalized by dividing by a single factor for that fiber before combining responses over the sample $(n=13)$. Solid lines and filled symbols show profiles (mean \pm SEM) for the standard stimulus and broken lines and open symbols show profiles (mean \pm SEM) for the comparison stimulus that was applied $0.5 \mathrm{~mm}$ proximal to the standard. Abscissae show the position of the standard along the $\mathrm{y}$ axis. A, Radius of spherical surface $1.92 \mathrm{~mm}$ (curvature $521 \mathrm{~m}^{-1}$ ). $B$, Radius $5.80 \mathrm{~mm}$ (curvature $172 \mathrm{~m}^{-1}$ ). 

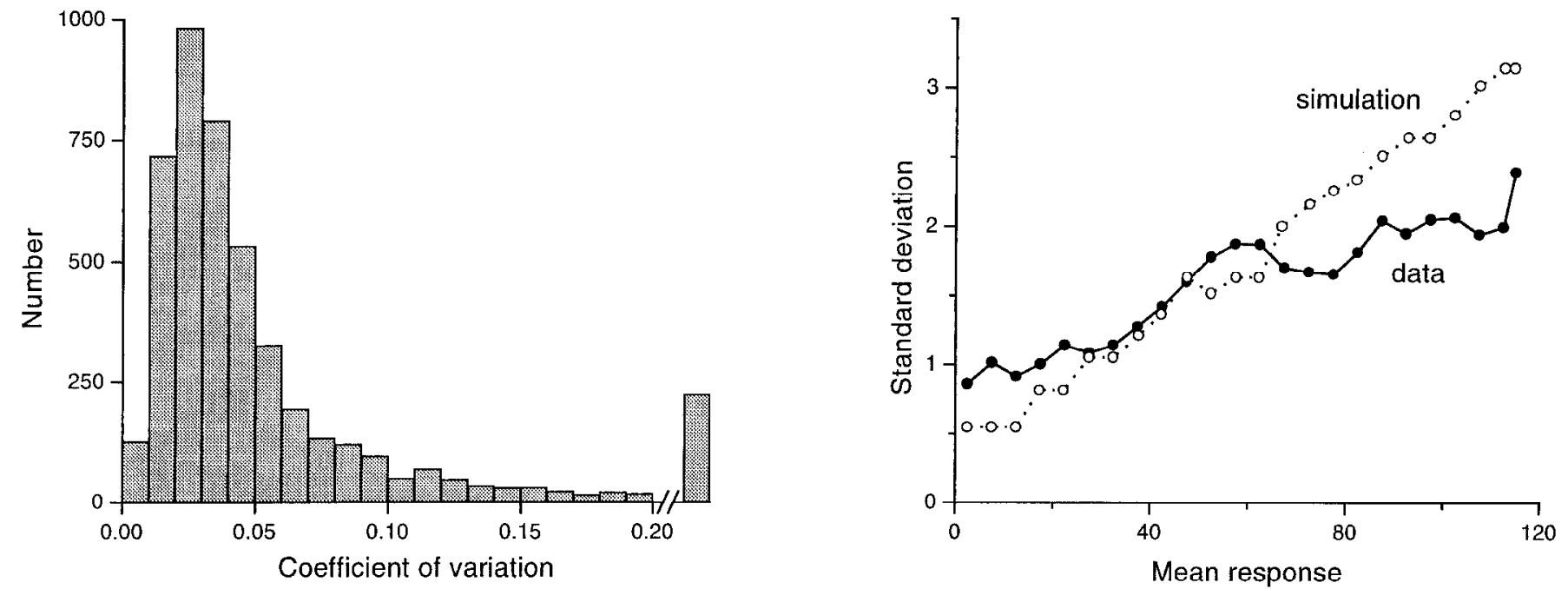

Figure 9. Variability of responses over the six trials in which the same stimulus conditions were repeated. Left, Histogram shows distribution of coefficients of variation (standard deviation divided by mean) of 4576 pairs of means and standard deviations. Bin width is 0.01 , last bin shows number exceeding 0.20. All responses (at all positions, for both spheres, for both conditions $S_{\mathrm{s}}$ and $S_{\mathrm{s}}$, and for all three differences in position between standard and comparison) are included for the 13 SAIs pooled in Figure 8. For each condition, the mean and standard deviation of the six repetitions were calculated. Right, The 4576 pairs were binned according to the value of their mean (bin width is 5). The average standard deviation in each bin is shown by the filled circles. Open circles show corresponding values for the simulation explained in Results.

drawn from 13 monkeys of two different species with a range of weights, the consistency of the profiles is remarkable.

\section{Analysis of variability}

Any meaningful comparison of human performance with neural responses must take account of the variability of the afferents' responses to repeated stimuli. This requires a quantitative analysis of variability to define its magnitude and to ascertain whether it varies with stimulus conditions. The means and standard deviations at each point in Figures 4-6 were calculated over the six trials where all stimulus parameters remained constant. The magnitude of the standard deviation appears to increase as the magnitude of the mean increases; or the coefficient of variation (standard deviation divided by mean) appears approximately constant. To confirm this observation, the coefficient of variation was examined for all responses, at all positions in the receptive field, and with all combinations of stimulus conditions, for all the SAIs used in the pooled data above. Scatter plots of mean versus standard deviation show that there is no difference in the pattern of variability for different curvatures or for different positions of the stimuli in the receptive field. The pattern is the same for standard and comparison stimuli, and is the same for $S_{\mathrm{s}}$ and $S_{\mathrm{d}}$ sequences. To quantify the magnitude of the variability all these data were pooled; the histogram showing the distribution of the coefficients of variation for these 4576 responses is shown in Figure 9 (left). Most of the values are closely distributed around the $3 \%$ mark. There is a tail with a small number of responses showing greater variability and a few, shown by the bar at the end of the histogram, had coefficients of variation exceeding $20 \%$. For those cases where the coefficient of variation was high, the mean response was low.

The reason why the variability appears to be high when the mean is low is clear from the following sequence of six responses taken from the data. The number of action potentials, in the first second of response, for the six repeated trials was 2 , $2,2,2,1$, and 2 . Thus, the mean response was 1.833 , the standard deviation 0.408 , and the coefficient of variation 0.223 . For the sequence $2,1,2,1,2$, and 3 , with the same mean, the coefficient of variation was 0.41 . The number of action potentials counted in a trial must be an integer, and the large coefficient of variation is a necessary adjunct to the quantal nature of our response measure. For example, a mean count of 1.833 action potentials can only result from integer counts, which are different in the six trials, and a large coefficient of variation is inevitable. In Figure 9 (right) the 4576 data points, each consisting of a mean and a standard deviation, have been grouped into bins according to the value of the mean. Filled symbols and the solid line show the average standard deviation in each bin. For comparison, open circles and the broken line show the results of a simulation as follows. For each bin, six random numbers were generated; the random number generator had a fixed mean (the value for that bin) and a coefficient of variation of $2.9 \%$. The six numbers were truncated to integers and their mean and standard deviation plotted by the open circles. The three characteristics of the data are also seen in the simulated data and are reflections of the random variation and quantization discussed above. These characteristics are as follows. (1) For higher means, standard deviation increases as the mean increases. (2) For lower means, the standard deviation is relatively greater. (3) There are some horizontal steps of unchanging standard deviation. Presumably, most strategies employed by the brain to discriminate the position of two stimuli will be dominated by the larger responses, and the apparent variability of the small responses is unlikely to be of significance. The significance of this detailed analysis is that it allows us to factor the variability into any neural code that we may wish to compare with psychophysical performance.

\section{Effect of analysis period}

So far, all neural activity has been defined by the number of action potentials evoked during the first second of response. We repeated the analysis for three additional time frames, namely $0.2,0.3$, and 0.5 seconds. The aims of this analysis are twofold; first, to examine how the profiles and the profile shifts are af- 


\section{A. $0.2 \mathrm{~s}$}

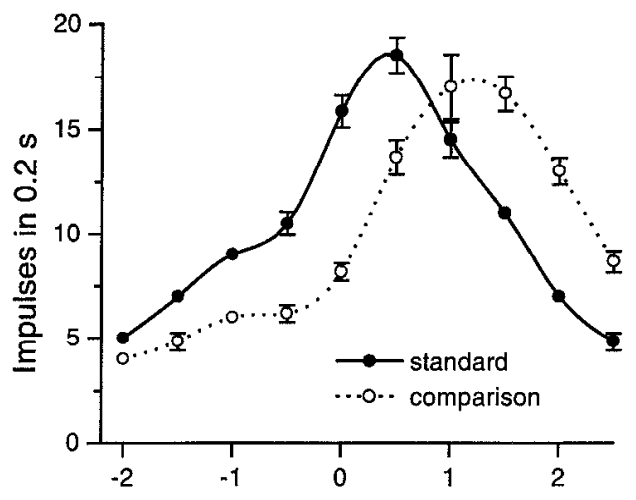

B. $0.3 \mathrm{~s}$

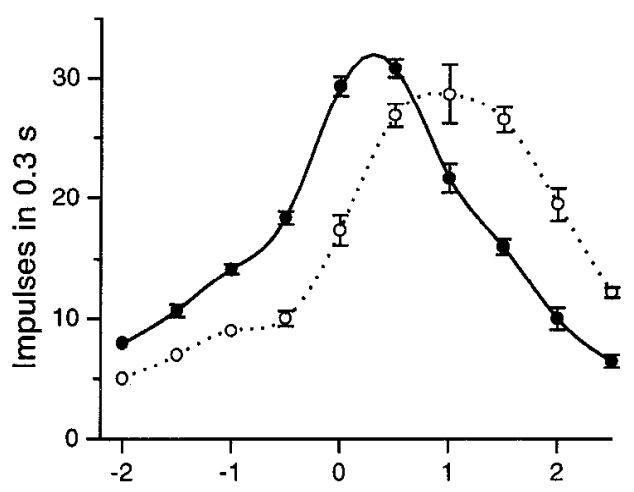

C. $0.5 \mathrm{~s}$

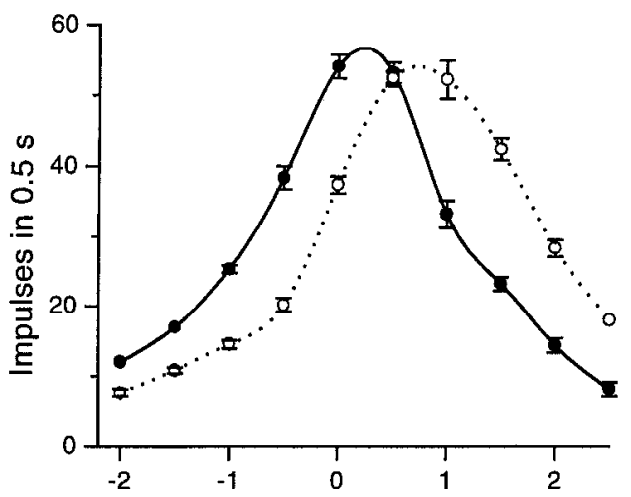

D. $1 \mathrm{~s}$

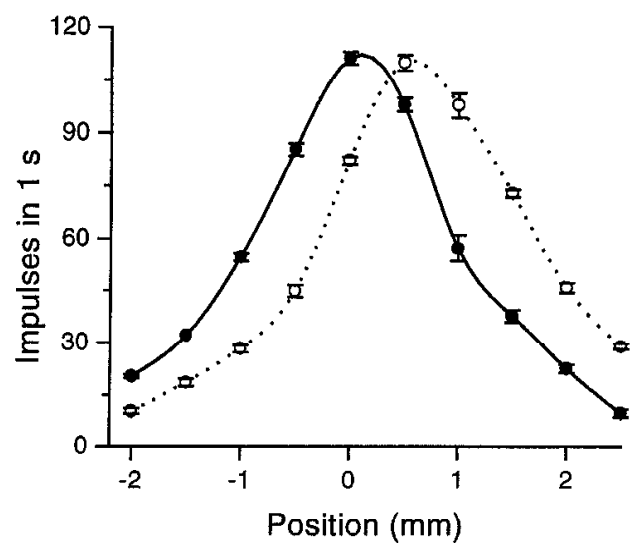

fected by the interval, and second, to examine how the variability of responses and thus the signal-to-noise ratios are affected. Profiles of responses defined by the number of action potentials occurring during the first $0.2,0.3,0.5$, and $1 \mathrm{sec}$ of response are shown for a typical SAI in Figure 10. The surface had a radius of $1.92 \mathrm{~mm}$ (curvature of $521 \mathrm{~m}^{-1}$ ) and the comparison stimulus was positioned $0.5 \mathrm{~mm}$ proximal to the standard. Profile shapes are similar for the four analysis intervals but, naturally, the magnitude of the response grows as the analysis interval increases. The rate of increase in response during the initial period (from 0.2 to $0.3 \mathrm{sec}$ ) is similar to that during the final period $(0.5$ to $1.0 \mathrm{sec})$ indicating that the dynamic component of response for this fiber was small. For all four intervals, the shift between profiles for the standard and the comparison stimuli is similar. Magnitudes of the standard deviations show that although the responses grew as the analysis interval increased, the standard deviations also grew. Thus, the coefficients of variation did not change much. The same result was obtained for the surface with a radius of $5.80 \mathrm{~mm}$ (curvature $172 \mathrm{~m}^{-1}$ ). To examine the consistency of this effect over all responses in all SAIs, the distributions of the coefficients of variation were compared for the four analysis intervals. The distributions for $0.2,0.3$, and $0.5 \mathrm{sec}$ shown in Figure 11 can be compared to the distribution for $1 \mathrm{sec}$ shown in Figure 9 (left). To reduce the contribution to Figure 11 from small responses with apparently high variability, responses were excluded if the count was less than four impulses. Clearly, the four distributions are similar. As the analysis interval decreases, the histograms show a slight bias towards larger values of the coefficient of variation and an increase in the first bin. The coefficient of variation indicates the signal-to-noise ratio or the resolution of the responses, and this did not change much with an increase in the analysis interval. This behavior of the response variability must be taken into account when considering neural codes defined over different time intervals.

For the fiber in Figure 10, the profile shapes were well established by $0.2 \mathrm{sec}$ and did not change much with time. Moreover, at all four intervals examined, the profiles were highly consistent from fiber to fiber. Figure 12 shows the spread of normalized profile shapes across the population of SAIs for responses summed over $0.2,0.3$, and $0.5 \mathrm{sec}$ for both spheres; these can be compared to the corresponding profiles at $1 \mathrm{sec}$ in Figure 8. For each fiber, a single normalizing factor was used; this was the value calculated from the responses over $1 \mathrm{sec}$. The variability among the afferents, indicated by the standard errors, is comparable at all four intervals. There is a suggestion of a slightly greater variability for the smaller intervals that could be, in part, because some fibers had a more prominent dynamic response than others. The profile shapes are similar al all four intervals.

Figure 10. Activity of a single SAI measured by the number of impulses during the first $0.2,0.3,0.5$, or $1.0 \mathrm{sec}$ of response $(A, B, C$, or $D$, respectively). Solid and broken lines show means $\pm \operatorname{SD}(n=6)$ for the standard and comparison stimuli, respectively. Spherical surface had a radius of $1.92 \mathrm{~mm}$ (curvature of $521 \mathrm{~m}^{-1}$ ). Abscissae show the position of the standard stimulus on the y-axis; the comparison was shifted $0.5 \mathrm{~mm}$ proximally. The response axis scales are different for different time frames; these were chosen to highlight the consistency of profile shapes and shifts, and the consistency of signal-to-noise ratios. 

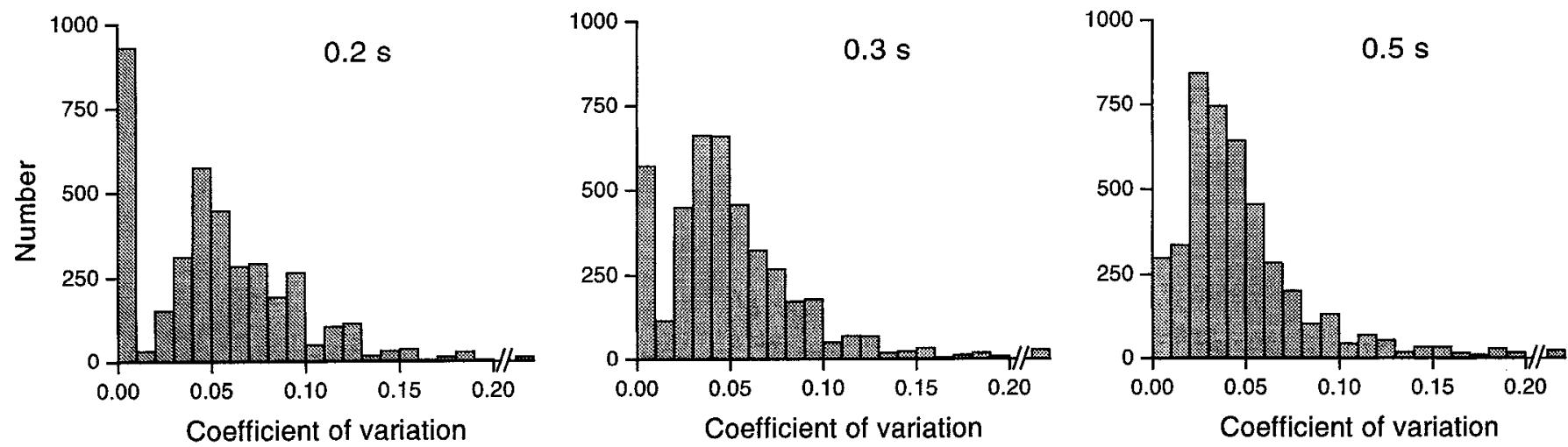

Figure 11. Histograms showing the distributions of the coefficients of variation for responses defined by the number of impulses in the first 0.2 , 0.3 , or $0.5 \mathrm{sec}$ (left, center, and right, respectively). Coefficients of variation were calculated over the six trials in which all conditions remained the same. Bin width is 0.01 , last bin shows number exceeding 0.20. Data include all of the 4576 points used in Figure 9 (responses for all conditions and at all positions for the 13 SAIs in the pool) for which the counts were four impulses or greater.

\section{$R A$ responses}

Ten of the rapidly adapting (Meissner) afferents sampled did not respond at all to our stimuli. For the remaining 11 , the responses were small but changed consistently with changes in the position of the stimulus. The responses of a single RA are shown in Figure 13, $A$ and $B$, for both spheres when the comparison stim- ulus was applied $0.5 \mathrm{~mm}$ proximal to the standard. Although, at any position, the mean number of impulses occurring during the first second of response is small (less than 10), the standard deviation over the six repetitions is correspondingly small. Comparing the profiles in Figure 13, $A$ and $B$, it is seen that the shapes are similar for both spheres. This is in keeping with our
A. $0.2 \mathrm{~s}$, radius $1.92 \mathrm{~mm}$

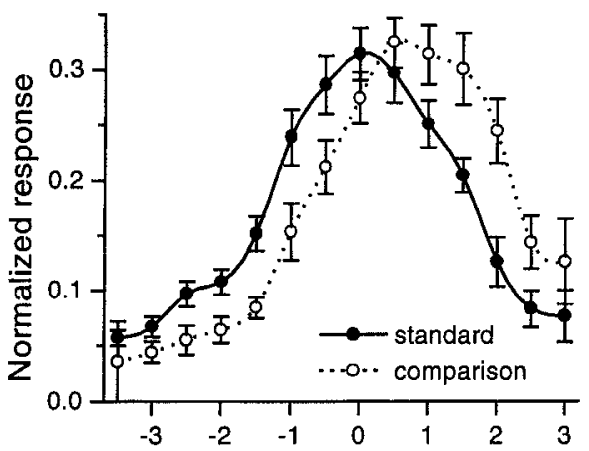

D. $0.2 \mathrm{~s}$, radius $5.80 \mathrm{~mm}$

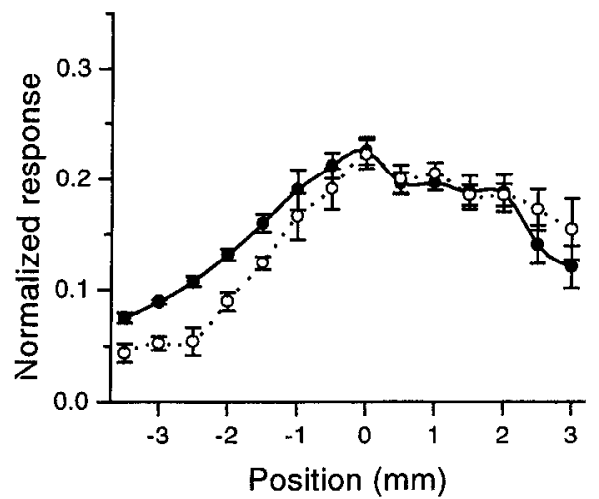

B. $0.3 \mathrm{~s}$, radius $1.92 \mathrm{~mm}$

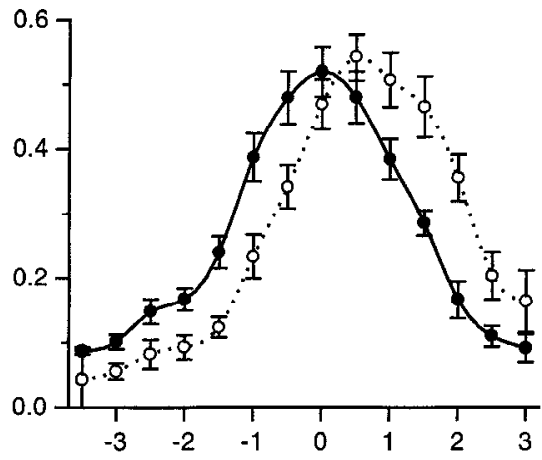

E. $0.3 \mathrm{~s}$, radius $5.80 \mathrm{~mm}$

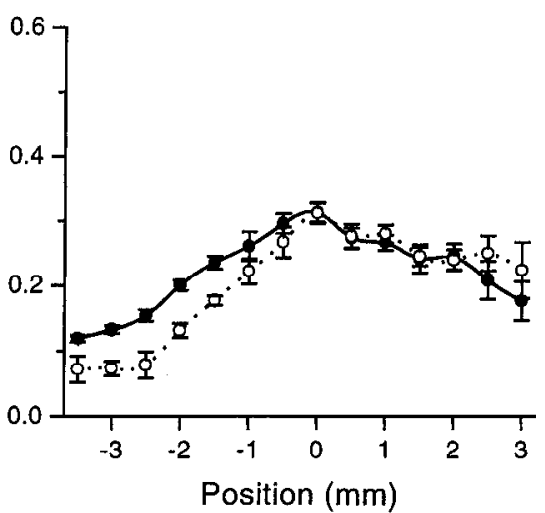

C. $0.5 \mathrm{~s}$, radius $1.92 \mathrm{~mm}$

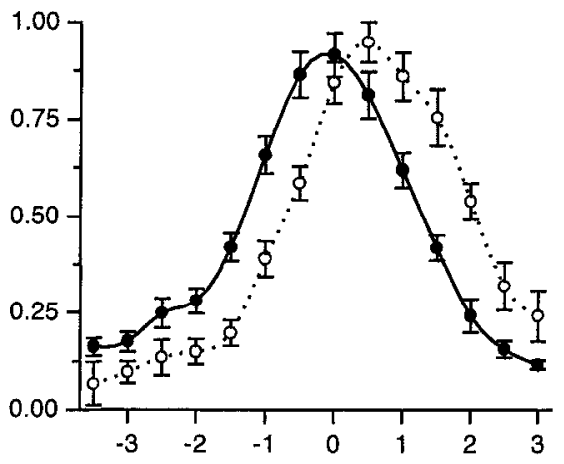

F. $0.5 \mathrm{~s}$, radius $5.80 \mathrm{~mm}$

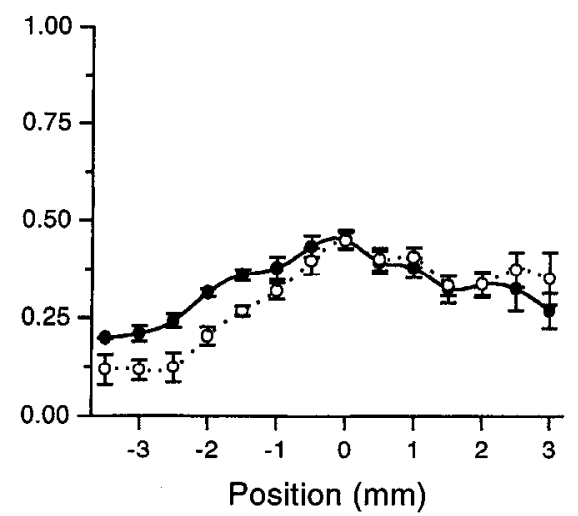

Figure 12. Normalized response profiles combined for the SAIs. Responses were defined by the number of impulses during the first 0.2 sec ( $A$ and $D), 0.3 \mathrm{sec}(B$ and $E$ ) or $0.5 \mathrm{sec}(C$ and $F)$. Means and standard errors are shown for the standard stimulus (solid lines), which was positioned at the abscissa values, and for the comparison (broken lines), which was positioned $0.5 \mathrm{~mm}$ proximal to the standard. The spherical surfaces had a radius of $1.92 \mathrm{~mm}$ (curvature $521 \mathrm{~m}^{-1}$ ) for $A-C$ and a radius of $5.80 \mathrm{~mm}$ (curvature $172 \mathrm{~m}^{-1}$ ) for $D-F$. Responses for the 13 SAIs (same fibers as in Fig. 8) were normalized before combining; for each fiber the normalizing factor used at all three intervals was the same as that used in Figure 8. Profiles are shown along the y-axis. For each time frame, scales on the response axes are the same for the two spheres, but they are different for different time frames. 


\section{A. Single RA (radius $1.92 \mathrm{~mm}$ )}

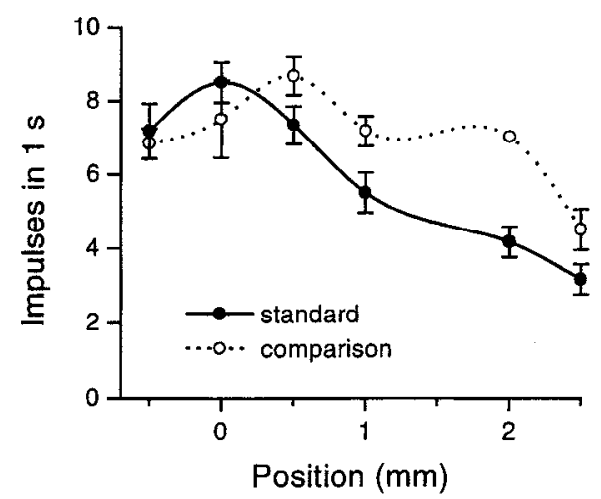

B. Single RA (radius $5.80 \mathrm{~mm}$ )

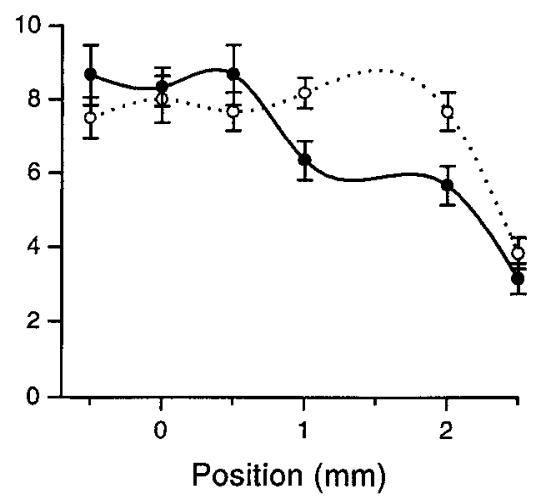

C. All RAs (radius $1.92 \mathrm{~mm}$ )

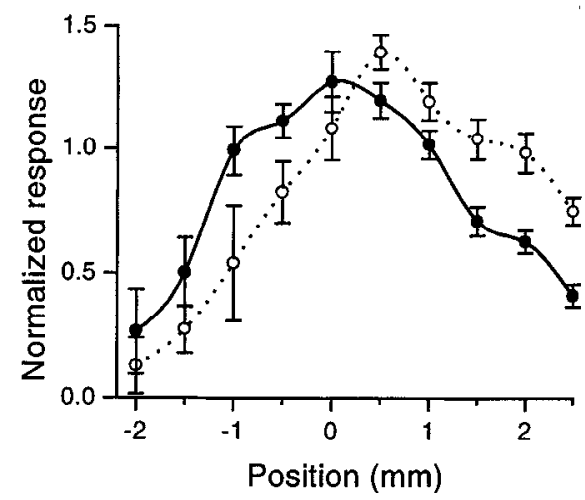

Figure 13. Response profiles of RAs when the comparison stimulus (broken line) was applied 0.5 mm proximal to the standard (solid line). Abscissae show the position of the standard along the $y$-axis. $A$ and $B$, For a single RA, the number of impulses during the first second of response are shown (mean $\pm \mathrm{SD}, n=6$ ). Radius of spherical surface was $1.92 \mathrm{~mm}$ (curvature $521 \mathrm{~m}^{-1}$ ) in $A$ and $5.80 \mathrm{~mm}\left(\right.$ curvature $172 \mathrm{~m}{ }^{-1}$ ) in $B$. $C$, Normalized responses (mean \pm SEM) of eight RAs combined; for each afferent, responses were divided by the average response of that afferent over five central positions before combining.

previous finding that the RA responses did not change systematically with changes in the curvature of the stimulus (Goodwin et al., 1995). However, the shift in the profiles with a shift in the position of the comparison stimulus is quite clear. A few of the responding RAs had larger responses than the RA in Figure 13, and a few had smaller responses, but in each case the standard deviations were small and the shift in the profiles was evident. The consistency of the shift can be seen in Figure 13C, where responses have been combined for eight RAs for which an extensive set of data was collected along the $y$-axis. As in the case of the pool of SAIs, the sensitivity of each individual afferent was first eliminated by normalizing responses by dividing by the average response over five central positions. The standard errors show the consistency among RAs. Thus, although half the RAs did not respond at all, and although the responses of the remainder were small in magnitude, there was reliable information present about differences in the positions of the standard and comparison stimuli.

\section{Discussion}

When spherically curved surfaces were applied passively to the fingerpad, humans were able to discriminate small differences in the positions of the stimuli. The average difference limens of $0.55 \mathrm{~mm}$ for the less curved sphere (radius $5.80 \mathrm{~mm}$, curvature $172 \mathrm{~m}^{-1}$ ) and $0.38 \mathrm{~mm}$ for the more curved sphere (radius 1.92 $\mathrm{mm}$, curvature $521 \mathrm{~m}^{-1}$ ) are considerably smaller than the classical "error of localization" which is nearly $2 \mathrm{~mm}$ (Dallenbach, 1932; Weinstein, 1968). In an experiment similar to ours, but using a more curved probe (radius $0.35 \mathrm{~mm}$, curvature 2857 $\mathrm{m}^{-1}$ ), Loomis (1979) obtained an average difference limen of $0.17 \mathrm{~mm}$. In his paradigm, subjects differentiated a displacement to the left of a central position from a displacement to the right, so that they may have adopted a strategy in which the left and right stimuli, separated by $0.34 \mathrm{~mm}$, were in fact distinguished. These differences, plus the variation amongst a relatively small number of subjects (the smallest difference limen amongst our subjects was $0.24 \mathrm{~mm}$ ), signify that our data and Loomis's arc consistent. There have been many other demonstrations of discriminative capacities significantly better than those predicted on the basis of classical measures like the two-point limen or the error of localization. A few examples are size and length discrimination (Jones and Vierck, 1973), motion of a stimulus on the fingertip (Loomis and Collins, 1978), tactile vernier acuity (Loomis, 1979), discrimination of gratings (Johnson and Phillips, 1981; Morley et al., 1983), and shape discrimination (LaMotte and Srinivasan, 1987). In many instances, the performance is also significantly hetter than that predicted on the basis of the sampling theorem. The estimated innervation density of the human fingerpad is 0.70 and 1.41 per $\mathrm{mm}^{2}$ for the SAIs and RAs, respectively (Johansson and Vallbo, 1979); similar innervation densities have been found in the monkey (Darian-Smith and Kenins, 1980). Assuming a square mosaic of innervation, the sampling theorem gives an upper limit of resolution of 0.42 and 0.59 cycles per $\mathrm{mm}$ (or a spatial period of 2.4 and $1.7 \mathrm{~mm}$ ) for the SAIs and RAs, respectively (Williams, 1986).

Acuity that is greater than that predicted by classical measures of spatial resolution or by the sampling theorem is often termed hyperacuity, and has been studied extensively in visual psychophysics (Westheimer, 1981). Hyperacuity should not be viewed as paradoxical, and it is important that the underlying neural mechanisms are clearly understood. In the visual system, there have been few experiments directly recording such neural responses. Recordings from cat retinal ganglion cells (Shapley and Victor, 1986) and monkey striate cortical cells (Parker and Hawken, 1985) have shown how these neurons respond to displacements of gratings much smaller than those suggested by their thresholds for resolving gratings. Similarly, responses of monkey retinal ganglion cells to small displacements of an edge (Lee et al., 1993) and of cat striate cortical cells to vernier acuity type stimuli (Swindale and Cynader, 1986) have been recorded. These results indicate how neural responses can resolve stimuli for which humans display hyperacuity. In the tactile system, most demonstrations of high resolution have not been accompanied by matching recordings of neural responses; a few exceptions are the discrimination of dot patterns (Johnson and Lamb, 1981; Phillips et al., 1992), of gratings (Phillips and Johnson, 1981; Sinclair and Burton, 1991), and of curved surfaces (Srinivasan and LaMotte, 1987).

In a previous study, we showed that the curvature of a spherical stimulus is reflected in the shape of the response profile in 
the SAI population (Goodwin et al., 1995). Our current data extend this observation by showing that when the position of the sphere on the skin changes, there is a corresponding shift in the profile of activity in the population of SAIs. It is clear from our data that (1) the size of the shift increases as the magnitude of the change in position increases; (2) measures of the shift (such as the volume between the original profile and the shifted profile) have larger magnitudes for the more curved sphere; (3) the shifts, and measures derived from them, are well above the noise level for position changes of $0.8 \mathrm{~mm}$ and get progressively closer to the noise level as the change in position decreases in magnitude; and (4) ultimately, the noise level will limit resolution. For the less curved sphere, this limit will occur at larger magnitude of change in position than for the more curved sphere. At this simple level, the neural responses match and explain the psychophysical performance. From inspection of the profile shifts, it is also obvious why such small differences can be resolved even though the SAI receptive field diameters, given by the distances over which our stimuli evoked responses, exceeded $6 \mathrm{~mm}$ for the more curved surface and were much greater for the less curved surface. In fact, it is this breadth of the response profiles that underlies the hyperacuity, since the shift is "measured" by receptors over an area in excess of $28 \mathrm{~mm}^{2}$, a significant number even with innervation densities as low as 0.7 per $\mathrm{mm}^{2}$.

\section{Response variability}

Two stimuli can only be resolved if some aspect of the corresponding neural responses differ and this difference exceeds the response variability. Most experiments concentrate on defining the responses, and little attention is paid to the variability. To assess the variability in our task, we used nearly identical time sequences of random stimuli in the psychophysical and the neurophysiological experiments. When response magnitude was defined by the number of action potentials during the first second of response, we found that an increase in mean response was accompanied by an increase in the standard deviation of the response. This was true both within a single fiber and across fibers. The coefficient of variation was closely distributed around a value of approximately $3 \%$. The importance of this result is highlighted by some quite different observations in the visual system. For small displacements of an edge in the receptive fields of monkey retinal ganglion cells, Lee et al. (1993) found that response variance remained approximately constant, despite a fourfold increase in mean response; coefficients of variation (determined from their Table 1) ranged from about 24 to $100 \%$. In contrast to this, Dean (1981) found that in anesthetized cats the variance of striate cortical cell responses to gratings increased approximately linearly with mean responses; variance was about 1.5 times the mean. In similar studies on cortical cells in anesthetized cats and monkeys (Tolhurst et al., 1983) and awake behaving monkeys (Vogels et al., 1989), response variance again increased approximately linearly with the mean and was about 2.8 and 2 times the mean, respectively. Two facts are striking. First, variability in mechanoreceptive afferents is much less than that in retinal ganglion cells or cortical neurons. Second, different neuron populations have different variability characteristics and unless the variability of the particular neurons being studicd is assessed, it is not possible to quantify their resolution. In our experiments, responses were suprathreshold. For many measures of resolution, such as visual detection of small movements of a grating, the neural basis is a threshold response above the noisy background (Shapley and Victor, 1986). In tasks such as the one we used, the neural basis is a comparison of two responses, each of which is considerably above the noise level; the issue here is whether the difference in the responses can be distinguished from the noise.

\section{Integration time}

Whether a change in the time over which responses are measured results in a change in resolution can only be determined after careful assessment of response variability. It is often noted that total responses increase when the time interval over which responses are integrated increases (LaMotte and Srinivasan, 1993; Goodwin et al., 1995). This is usually taken, implicitly or explicitly, to mean an increase in resolution with an increase in integration time, but this is only true if signal-to-noise ratios have increased. For our stimuli, increasing the analysis time increased the variability of the responses as well as their mean values. Thus, increasing the integration time from 0.2 to $1 \mathrm{sec}$ resulted in only a minor increase in signal-to-noise ratios or resolution. Unfortunately, we do not have psychophysical data comparing human performance for $0.2 \mathrm{sec}$ of contact with that for $1 \mathrm{sec}$ of contact. However, in our psychophysical experiments, it was often noticed that subjects responded well before the stimulus was lifted off the skin; that is, in less than $1 \mathrm{sec}$.

\section{Quantitative assessment}

At a superficial level, our neural data match and explain the psychophysical performance. A more rigorous comparison requires a more detailed analysis taking into account the following. First, specific measures that may be extracted from the population response must be defined; in Figure 6, we referred to the area between the response profiles along one axis, but there are other possibilities. Second, the innervation density of the afferents is critical; profiles akin to those in Figure 8 are sampled at a rate determined by afferent density rather than every $0.5 \mathrm{~mm}$, which is the experimental sampling rate. Third, sensitivity will vary among the afferents; this will "distort" the population profiles suggested by Figure 8 . Fourth, the statistical variation in selected response measures must be accounted for. We now have all the information needed for such modeling, including the discrimination of curvature as well as the discrimination of position, but it is a substantial analysis beyond the scope of this article and will be reported elsewhere.

\section{Rapidly adapting responses}

Although the responses of the RAs were small compared to that of the SAIs, the variability was correspondingly small so that RAs relayed information about small changes in the position of the stimulus. However, half the RAs did not respond at all, and it is not obvious how this would affect the population response. In the unlikely event of every alternate RA responding for both positions of the stimulus, the effective innervation density would be halved, but if patches of RAs did not respond, the effect would be marked and unpredictable. It is difficult to see how the brain could use this information, or why it should be used when such a clear signal is present in the SAI population. It is possiblc that the RA responses and the SAI responses may somehow be used together to enhance the resolution of the SAIs. We did not record from pacinians, as most of these do not respond to our stimuli (Goodwin et al., 1995). 


\section{References}

Dallenbach KM (1932) A comparative study of the errors of localization on the finger-tips. Am J Psychol 44:327-331.

Darian-Smith I, Kenins P (1980) Innervation density of mechanoreceptive fibres supplying glabrous skin of the monkey's index finger. J Physiol (Lond) 309:147-155.

Dean AF (1981) The variability of discharge of simple cells in the cat striate cortex. Exp Brain Res 44:437-440.

Goodwin AW, Morley JW (1987) Sinusoidal movement of a grating across the monkey's fingerpad: representation of grating and movement features in afferent fiber responses. J Neurosci 7:2168-2180.

Guodwin AW, Johı KT, Marceglia AH (1991) Tactile discrimination of curvature by humans using only cutaneous information from the fingerpads. Exp Brain Res 86:663-672.

Goodwin AW, Browning AS, Wheat HE (1995) Representation of curved surfaces in responses of mechanoreceptive afferent fibers innervating the monkey's fingerpad. J Neurosci 15:798-810.

Johansson RS, Vallbo AB (1979) 'Tactile sensibility in the human hand: relative and absolute densities of four types of mechanoreceptive units in glabrous skin. J Physiol (Lond) 286:283 300.

Johnson KO (1980) Sensory discrimination: decision process. I Neurophysiol 43:1771-1792.

Johnson KO, Lamb GD (1981) Neural mechanisms of spatial tactile discrimination: neural patterns evoked by Braille-like dot patterns in the monkey. J Physiol (Lond) 310:117-144.

Johnson KO, Phillips JR (1981) Tactile spatial resolution. I. Two-point discrimination, gap detection, grating resolution, and letter recognition. J Neurophysiol 46:1177-1191.

Jones MB, Vierck CJ (1973) Length discrimination on the skin. Am J Physiol 86:49-60.

LaMotte RH, Srinivasan MA (1987) Tactile discrimination of shape: responses of slowly adapting mechanoreceptive afferents to a step stroked across the monkey fingerpad. J Neurosci 7:1655-1671.

LaMotte RH, Srinivasan MA (1993) Responses of cutaneous mechanoreceptors to the shape of objects applied to the primate fingerpad. Acta Psychol (Amst) 84:41-51.

Lee BB, Wehrhahn C, Westheimer G, Kremers J (1993) Macaque ganglion cell responses to stimuli that elicit hyperacuity in man: detection of small displacements. I Neurosci 13:1001-1009.

Loomis JM (1979) An investigation of tactile hyperacuity. Sensory Proc 3:289-302.

Loomis JM, Collins CC (1978) Sensitivity to shifts of a point stimulus: an instance of tactile hyperacuity. Percept Psychophys 24:487-492.
Morley JW, Goodwin AW, Darian-Smith I (1983) Tactile discrimination of gratings. Exp Brain Res 49:291-299.

Parker A, Hawken M (1985) Capabilities of monkey cortical cells in spatial-resolution tasks. J Opt Soc Am 2:1101-1114.

Phillips JR, Johnson KO (1981) Tactile spatial resolution. II. Neural representation of bars, edges, and gratings in monkey primary afferents. J Neurophysiol 46:1192-1203.

Phillips JR, Johansson RS, Johnson KO (1992) Responses of human mechanoreceptive afferents to embossed dot arrays scanned across fingerpad skin. J Neurosci 12:827-839.

Shapley R, Victor J (1986) Hyperacuity in cat retinal ganglion cells. Science 231:999-1002.

Sinclair RJ, Burton H (1991) Tactile discrimination of gratings: psychophysical and neural correlates in human and monkey. Somatosens Mot Res 8:241-248.

Srinivasan MA, LaMotte RH (1987) Tactile discrimination of shape: responses of slowly and rapidly adapting mechanoreceptive afferents to a step indented into the monkey fingerpad. J Neurosci 7:16821697.

Swindale NV, Cynader MS (1986) Vernier acuity of neurons in cat visual cortex. Nature 319:591-593.

Talbot WH, Darian-Smith I, Kornhuber HH, Mountcastle VB (1968) The sense of flutter-vibration: comparison of the human capacity with response patterns of mechanoreceptive afferents from the monkcy hand. J Neurophysiol 31:301-334.

Tolhurst DJ, Movshon JA, Dean AF (1983) The statistical reliability of signals in single neurons in cat and monkey visual cortex. Vision Res 23:775-785.

Vallbo AB, Johansson RS (1984) Properties of cutaneous mechanoreceptors in the human hand related to touch sensation. Hum Neurobiol 3:3-14.

Vogels R, Spileers W, Orban GA (1989) The response variability of striate cortical neurons in the behaving monkey. Exp Brain Res 77: $432-436$.

Weinstein S (1968) Intensive and extensive aspects of tactile sensitivity as a function of body part, sex, and laterality. In: The skin senses (Kenshalo DR, ed), pp 195-222. Springfield, IL: Thomas.

Westheimer G (1975) Visual acuity and hyperacuity. Invest Ophthalmol Vis Sci 14:570-572.

Westheimer G (1981) Visual Hyperacuity. Prog Sensory Physiol 1:130 .

Williams DR (1986) Seeing through the photoreceptor mosaic. Trends Neurosci 9:193-198.

Zigler MJ (1935) The experimental relation of the two-point limen to the error of localization. J Gen Psychol 13:316-332. 